\title{
Genetic structure in patchy populations of a candidate foundation plant: a case study of Leymus chinensis (Poaceae) using genetic and clonal diversity
}

Jian Guo ${ }^{1,7}$, Christina L. Richards ${ }^{2,3}$, Kent E. Holsinger ${ }^{4}$, Gordon A. Fox ${ }^{2,5}$, Zhuo Zhang ${ }^{6}$, Chan Zhou $^{1,8}$

${ }^{1}$ School of Life Science, Liaoning University, Shenyang 110036, P.R. China

${ }^{2}$ Department of Integrative Biology, University of South Florida, Tampa, FL 33620, USA

${ }^{3}$ Plant Evolutionary Ecology group, University of Tübingen, D-72076 Tübingen, Germany

${ }^{4}$ Department of Ecology and Evolutionary Biology, University of Connecticut, U-3043, Storrs, Connecticut 06269, USA

${ }^{5}$ Department of Biology, University of New Mexico, Albuquerque, NM 87131, USA

${ }^{6}$ School of Life Science and Bioengineering, Shenyang University, Shenyang 110044, P.R.

China

${ }^{7}$ School of Environmental Engineering, Xuzhou University of Technology, Xuzhou 221018,

P.R. China

${ }^{8}$ Author for correspondence (e-mail: zhou_yanhan@163.com)

RUNNING HEAD: Clonal diversity in a grassland foundation plant species. 


\begin{abstract}
PREMISE: The distribution of genetic diversity on the landscape has critical ecological and evolutionary implications. This may be especially the case on a local scale for foundation plant species since they create and define ecological communities, contributing disproportionately to ecosystem function.
\end{abstract}

METHODS: We examined the distribution of genetic diversity and clones, which we defined first as unique multi-locus genotypes (MLG), and then by grouping similar MLGs into multilocus lineages (MLL). We used 186 markers from inter-simple sequence repeats (ISSR) across 358 ramets from 13 patches of the foundation grass Leymus chinensis. We examined the relationship between genetic and clonal diversities, their variation with patch-size, and the effect of the number of markers used to evaluate genetic diversity and structure in this species.

RESULTS: Every ramet had a unique MLG. Almost all patches consisted of individuals belonging to a single MLL. We confirmed this with a clustering algorithm to group related genotypes. The predominance of a single lineage within each patch could be the result of the accumulation of somatic mutations, limited dispersal, some sexual reproduction with partners mainly restricted to the same patch, or a combination of all three.

CONCLUSIONS: We found strong genetic structure among patches of L. chinensis. Consistent with previous work on the species, the clustering of similar genotypes within patches suggests 
that clonal reproduction combined with somatic mutation, limited dispersal, and some degree of sexual reproduction among neighbors causes individuals within a patch to be more closely related than among patches.

KEY WORDS: asexual reproduction; clonal variation; foundation species; ISSR; Chinese grassland; somatic mutation. 


\section{INTRODUCTION}

Genetic diversity provides the raw material for evolutionary change and thus the potential to adapt to changing environments (Frankel and Soulé, 1981), but how genetic diversity is distributed on the landscape has critical ecological and evolutionary implications (Slatkin, 1987;

Whitham et al., 2006; Hughes et al., 2008; Sork et al., 2013; Hughes and Lotterhos, 2014).

Although the theoretical importance of genetic diversity for the long-term persistence of populations is well understood, we have little information about the fine-scale distribution of genetic variation in natural populations (Morris et al., 2011; Gray et al., 2014; Hughes and Lotterhos, 2014). This is true even for species that are quite common or dominate a landscape. According to Ellison (2019), 50 years of research has identified that there are "foundation species" that 1) dominate a community of species (numerically and in overall size), 2) determine the diversity of associated taxa through non-trophic interactions, and 3) modulate fluxes of nutrients and energy in their ecosystem. Although it is challenging to document that a candidate species meets all of the requirements to be considered a foundation species, there is growing recognition that protection and restoration of these species can disproportionately maintain habitat integrity and ecosystem resilience (Keith et al., 2017; Ellison, 2019; Qiao et al., 2021). The genetic make-up of foundation plant species can be particularly important on a local scale since these species create and define ecological communities and contribute disproportionately to 
ecosystem function (Ellison et al., 2005; Hughes and Lotterhos, 2014; Lau et al., 2016; Keith et al., 2017; Whitham et al., 2020).

Grassland species are often able to spread clonally through apomictic or vegetative means. In spite of their ecological importance in these systems, clonal organisms are thought to be limited in evolutionary potential since they lack the ability to generate variation and purge deleterious mutations through recombination (Lynch et al., 1993; Holsinger, 2000; but see Verhoeven and Preite, 2014). However, most clonal plants have some level of sexual reproduction (Richards, 1986), and an estimated two-thirds of temperate plant species are thought to be capable of clonal propagation (Van Groenendael and De Kroon, 1990; Klimes et al., 1997; Schoen and Schultz, 2019). Genetic diversity at marker loci is commonly measured as the proportion of polymorphic loci and as expected heterozygosity among all individuals, while clonal (or genotypic) diversity can be defined by the number of unique genotypes (evaluated across multiple loci) within a group (Sole et al., 2004; Arnaud-Haond et al., 2007; Reynolds et al., 2012). Although functional genetic variation is most important for understanding evolutionary responses (Texeira and Huber, 2021), the distribution of ramets per clone provides information on how genetic diversity is structured and therefore how natural selection might be constrained (Frankel, 1970; Widén et al., 1994; Arnaud-Haond et al., 2007). Hence, when considering how genetic diversity is spatially structured in plants that can spread vegetatively, it 
is useful to consider the abundance and distribution of ramets per clone in addition to population levels of genetic diversity. When conserving or restoring communities of such clonal plants, the relationship between genetic diversity and clonal diversity is an important consideration. The two metrics capture different aspects of genetic structure that may be related to the adaptive capacity and long-term viability of these plants (Schaal et al., 1991; Holsinger, 2000; Han et al., 2007; Reynolds et al., 2012). Several studies have found that populations of clonal species are generally just as diverse as non-clonal species (Ellstrand and Roose, 1987; Widén et al., 1994; Franks et al., 2004; Houliston and Chapman, 2004; Keeler et al., 2002; Richards et al., 2004; Raabová et al., 2015), but there are important exceptions particularly in the context of range expansion or invasive species (Ainouche et al., 2004, 2009; Gao et al., 2010; Richards et al., 2012; Zhang et al., 2016; Mounger et al., 2021).

Clonal foundation plant species can form vast monospecific stands, but they may also be found in patches that vary greatly in size in the wild due to disturbance, patch age and the favorability of conditions (Daehler and Strong, 1994; Davis et al., 2004; Travis and Hester, 2005; Morris et al., 2011; Gray et al., 2014; Hughes and Lotterhos, 2014). Genetic structure in clonal plants partly results from the fact that a single individual that establishes by seed or vegetative organ can produce many individual ramets through clonal propagation (Richards, 1986;

Lehmann, 1997; Chen et al., 2006), which can exhibit a patchy, interdigitated distribution (Zhou 
et al., 2003; Franks et al., 2004; Richards et al., 2004). Ramets that belong to a given genet can also accumulate mutations and appear to be new MLGs (Schoen and Schulz, 2019), even though functionally they could be more appropriately considered the same lineage (hence MLL). This can lead to different patterns of genetic diversity in older patches. In one scenario, patches may accumulate a large number of lineages, which each expand to result in a large patch area with high clonal diversity (Raabová et al., 2015). On the other hand, patches may have limited recruitment, or competitively superior lineages may produce many ramets through vegetative propagation to enlarge the patch area such that long-lived populations may be characterized by fewer genotypes (Sakai, 1995; Gardner and Mangel, 1999; Travis and Hester, 2005). However, detecting these different patterns of clonal structure can be complicated by outcrossing between closely related lineages, as well as the fact that somatic mutations may accumulate and provide an important source of variation in older lineages (Schoen and Schultz, 2019).

Despite the fact that most of evolutionary theory and models are based on sexually reproducing, genetically distinct individuals, with the rapidly escalating application of molecular approaches to ecological questions, researchers have developed some sophisticated approaches to more accurately estimate genetic and clonal diversity in organisms that have a mix of both types of reproduction (Arnaud-Haond et al., 2007; Kamvar et al., 2014, 2015). In particular, it is useful to define a multi-locus lineage (MLL) as a set of distinct multi-locus genotypes (MLGs) 
that are more closely related to one another than any of them are to other genotypes. Identifying unique MLLs requires enough polymorphic markers to provide discriminating power to differentiate closely related genotypes, but also requires identification of scoring errors that will make estimates of relationships inaccurate (Douhovnikoff and Dodd, 2003; reviewed in ArnaudHaond et al., 2007). In the past, researchers did not distinguish between genetically distinct individuals (MLGs) and MLLs (e.g., Franks et al., 2004; Richards et al., 2004). As the number of markers has increased, however, allowing more closely related MLGs to be distinguished from one another, it has also become more important to distinguish among degrees of relationship (Douhovnikoff and Dodd, 2003; Arnaud-Haond et al., 2007; Kamvar et al., 2015). A common approach to identifying degrees of relationship is to use clustering algorithms to identify MLLs, groups of genotypes that are more similar to one another than any of them is to another genotype (Kamvar et al., 2015).

In this study, we examined the relationship between patch size and genetic structure in patches of the clonal plant Leymus chinensis, a dominant perennial grass species in the Chinese steppe zone (Li et al., 2014; Zhou et al., 2014; Zhou et al., 2015). While this species has not been formally referred to as a foundation species, several authors have argued that L. chinensus is one of the most important dominant species of the undisturbed mature community native to the Inner Mongolia grasslands (Bai et al., 2004, 2010; Tong et al., 2004; Meng et al., 2019). Previous work 
comparing undisturbed mature grassland communities to severely degraded communities showed that $L$. chinensis had the highest relative biomass and lowest interannual variability of biomass production among all species in the undisturbed system which contributed to ecosystem stability (Bai et al., 2004). These studies suggest that L. chinensis is a good "candidate foundation species" which will require further validation (sensu (Qiao et al., 2021)). Only a few studies have examined genetic variation in L. chinensis, and they have focused on large geographical scales, finding significant variation among different regions of China (Wang et al., 2005) and significantly higher genetic diversity in eastern than in western sites along a longitudinal gradient in northeast China (Yuan et al., 2016). However, there is very little information about how the genetic diversity of L. chinensis is distributed among different patches on a micro-geographical scale (e.g., Hong, 2013) or how genetic diversity is related to ecosystem resilience in this system (e.g., Bau et al., 2004). This knowledge is essential for managers to formulate the best strategy for conservation and restoration of this important grassland species. The structure of genetic diversity in patchy populations of $L$. chinensis could be an unappreciated but important component of successful restoration of degraded lands that were once dominated by this species (Bai et al., 2004; Tong et al., 2004). Using a large number of Inter Simple Sequence Repeat (ISSR) markers, we addressed the following questions: (1) What is the relationship between genetic and clonal diversity? (2) Do larger patches have higher levels of genetic and clonal diversity? (3) Is genetic or clonal structure correlated with patch size? Because we used a large 
number of ISSR markers, we also explored the technical question of how the number of markers used affects conclusions about the numbers of MLLs, as well as whether patches are predominantly composed of MLGs more closely related to one another than to MLGs in other patches.

\section{MATERIALS AND METHODS}

Study species - Leymus chinensis is a dominant tetraploid perennial grass species in the Chinese steppe zone (Li et al., 2014; Zhou et al., 2014; Zhou et al., 2015) occurring on a total area in Asia of approximately 420,000 $\mathrm{km}^{2}$, of which $220,000 \mathrm{~km}^{2}$ are located in China (Inner-Mongolia and Ning Xia Investigation and Survey Team of the Chinese Academy of Sciences 1985). Leymus chinensis is a significant forage grass, with high nutrient value, good palatability and high aboveground biomass production (Zhu, 2004; Bai et al., 2009). However, vast acreage of the species has suffered from overgrazing which has led to "steppe degradation" (Bai et al., 2004; Tong et al., 2004). As an ecologically and economically important foundation grass, L. chinensis has received considerable attention in recent years (Bai et al., 2004; Tong et al., 2004; Zhu, 2004).

A large number of investigations have focused on growth (Li et al., 2014; Liu et al., 2011), reproduction (Bai et al., 2009) and physiology (Xu and Zhou, 2007) of the species. Leymus chinensis can successfully survive under stressful conditions, such as drought, salinity, alkalinity, and depleted organic matter. The species possesses the capacity for both vegetative propagation 
through rhizome fragmentation and sexual reproduction by seeds (Bai et al., 2009). In natural habitats, L. chinensis appear in differently sized patches, and a single genet can give rise to multiple ramets and extensively spreading clones (Yang et al., 2006). Vegetative reproduction is thought to be more common than sexual reproduction (Wang, 2001; Zhang et al., 2005), but the actual contributions of these two modes of reproduction is unknown. When it reproduces sexually, L. chinensis is partially self-incompatible (with self-pollen leading to seed set in 5-10\% of experimental pollinations, as compared with 60-84\% of non-self-pollen) (Zhang et al., 2004); and as in other grasses the SI system is gametophytic (Chen et al., 2019).

Study sites - This study was carried out at the Grassland Ecological Research Station of

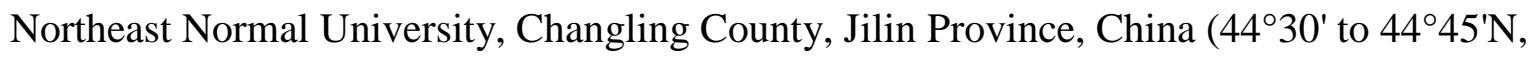
$123^{\circ} 31^{\prime}$ to $\left.123^{\circ} 56^{\prime} \mathrm{E}\right)$. This area is in the northeast of the Chinese steppe zone. The grassland has low-lying topography, and a typical mesothermal monsoon climate with hot, rainy summers and cold, arid winters. Mean annual precipitation ranges from 300 to $450 \mathrm{~mm}$, more than $60 \%$ of which occurs from June to September. Mean annual temperature ranges from 4.6 to $6.4^{\circ} \mathrm{C}$ and the frost-free period lasts for 130 to 165 days (Guo et al., 2020). The soil is a mixed salt-alkali meadow steppe (Salid Aridisol, US Soil Taxonomy) with 29\% sand, $40 \%$ silt and $31 \%$ clay (top $10 \mathrm{~cm}$ ) (Zhu, 2004). Grazing of this area was prohibited beginning in September 1995. Other 
plant species typically co-occuring with L. chinensis include Phragmites communis, Kalimeris integrifolia, Carex duriuscula, Calamagrostis epigeios, and Chloris virgata.

Sampling design - We collected tissue samples in August 2010, when the plants were vigorously growing. Thirteen L. chinensis patches were selected haphazardly within an area of $2000 \mathrm{~m}^{2}$. Patches were defined naturally (by the space occupied by L. chinensis locally), and all patches were separated from others by at least $200 \mathrm{~m}$. Patches ranged in area between $2.8 \mathrm{~m}^{2}$ and $166.8 \mathrm{~m}^{2}$ We collected vegetative tillers of $L$. chinensis at distances of $0,0.5,1,2,4,8$, and $16 \mathrm{~m}$ from the center of each patch (or to the edge of the patch, whichever came first) in each of eight directions (N, S, E, W, NE, NW, SE, SW). Consequently, we sampled between 17-35 ramets from each patch (Table 2). The vegetative ramets of L. chinensis were harvested at the ground surface and each ramet was put into a zip-lock plastic bag with silica gel to preserve the tissue. We transported all samples to the laboratory and stored them at $-20^{\circ} \mathrm{C}$.

DNA extraction - For each sample, we performed extractions of total genomic DNA using a modified CTAB method (Doyle and Doyle, 1987). We ground tissue of dried young leaves $(0.025$ to $0.030 \mathrm{~g})$ to a fine powder in liquid nitrogen with pure quartz sand in a $2 \mathrm{ml}$ centrifuge tube. Then, we added $400 \mu$ l of extraction buffer [ $2 \%$ cetyl trimethyl ammonium bromide (CTAB); $100 \mathrm{mM}$ Tris (pH=8.0); $20 \mathrm{mM}$ ethylenediamine tetra-acetic acid (EDTA); 0.5\% $\mathrm{NaHSO}_{3} ; 1.4 \mathrm{M} \mathrm{NaCl} ; 1 \%$ polyvinylpyrrolidone (PVP); $\% \beta$-mercaptoethanol] to the ground 
sample and incubated at $65^{\circ} \mathrm{C}$ for 30 minutes. We performed two rounds of extraction with 400 $\mu \mathrm{l}$ of tris-saturated phenol. After centrifugation, we performed two more rounds of extraction with chloroform: isoamyl alcohol (24:1) and precipitated the DNA with $200 \mu$ of 5 M potassium acetate and $1 \mathrm{ml}$ of $100 \%$ ice-cold ethanol. The precipitate was washed with $70 \%$ ethanol twice, dried, and resuspended in 100ul of 1×Tris-EDTA (TE) buffer (Shimizu et al., 2002). We estimated the quantity of DNA by comparing band intensities with known amounts of lambda DNA on $0.8 \%$ agarose gel. DNA quality was checked by spectrophotometry at $260 \mathrm{~nm}$ and 280 nm. All the DNA samples were stored at $-20^{\circ} \mathrm{C}$ for ISSR analysis.

ISSR amplification - Twenty-one primers from the Biotechnology Laboratory, University of British Columbia (UBC set no.9) were synthesized by Sangon (Shanghai Sangon Biological Engineering Technology and Services Co. Ltd, Shanghai, China) for initial screening.

Eventually, we selected 11 primers that produced clear bands with good reproducibility and high polymorphism (Table 1).

We performed ISSR amplification in a volume of $10 \mu$ containing 10 ng template DNA, $1 \mu 1$ 10×PCR Buffer, $20 \mathrm{mM} \mathrm{MgCl} 2,10 \mathrm{mM}$ dNTPs, $1.5 \mu \mathrm{M}$ primer, and $0.4 \mathrm{U}$ of Taq DNA polymerase. All amplifications were carried out in a Veriti 96 Well Thermal Cycler (Applied Biosystems, USA). For primers $6,10,13,807,818,827,834,842,848$ and 855 , the PCR reaction procedure was as followed: an initial denaturation step of 2 min at $94^{\circ} \mathrm{C}, 35$ cycles of 1 
min at $94^{\circ} \mathrm{C}, 1 \mathrm{~min}$ at respective $T_{\mathrm{m}}$ values (Table 1 ), and $1 \mathrm{~min}$ at $72^{\circ} \mathrm{C}$, and a final extension step of $10 \mathrm{~min}$ at $72^{\circ} \mathrm{C}$. For primer 811 , the amplifications began with initial denaturation for 5 min at $94^{\circ} \mathrm{C}$, followed by 45 cycles of $30 \mathrm{~s}$ at $94^{\circ} \mathrm{C}, 45 \mathrm{~s}$ at $56^{\circ} \mathrm{C}$, and $2 \mathrm{~min}$ at $72^{\circ} \mathrm{C}$, and a final extension at $72^{\circ} \mathrm{C}$ for $5 \mathrm{~min}$. The amplification products obtained were separated on $2 \%$ agarose gels containing $0.1 \%$ ethidium bromide at $120 \mathrm{~V}$ for $2 \mathrm{~h}$, and photographed under UV light. The molecular weight of every amplified band was evaluated by using the DL 2000 Marker (Beijing Dingguo Changsheng Biotechnology Co. Ltd, Beijing, China). Each reaction was repeated twice for each sample with each primer. Only those primers which generated reproducible bands in both reactions for each sample were used for the data analysis.

Data analysis - Only fragments that were distinct, reproducible, and well-resolved were included in the analysis. We scored all amplified fragments as present (1) or absent (0) by comparing size with external standards.

We used R software, version 3.43 (R Core Team, 2017), for all data analyses unless otherwise noted. We examined graphically the richness and distribution of markers within each of the 13 patches (Figure 1, Table 2), and generated a heatmap of marker frequencies using the heatmap. 2 function in the R library gplots. For purposes of our analysis we define a multilocus lineage as a set of distinct genotypes more closely related to one another than any of them are to other genotypes. To assess the number of multilocus lineages (MLLs) we used three approaches: 
(1) we examined the density plot of numbers of marker differences between all pairs of sampled individuals, to seek an intermediate minimum (sensu Douhovnikoff and Dodd, 2003; Kamvar et al., 2014, 2015). If the distribution of markers is the consequence of differentiation between MLLs (to the right) and somatic mutation within lineages plus measurement error (to the left), the minimum provides a criterion for distinguishing genotypes that belong to the same MLL (those with differences to the left of the minimum,) and those belonging to different MLLs (those with difference to the right). Specifically, following Kamvar et al. (2014, 2015) we calculated the Prevosti distances between all pairs of samples and used these distances to estimate a threshold to distinguish within-lineage from among-lineage differences. (2) we assigned ramets to MLLs using the infinite alleles model in GenoDive (Meirmans and Van Tienderen, 2004; Meirmans, 2020), using cutoffs between 14 and 42 marker differences (based on the approximate location of a minimum in the density plot) to determine clonality. We then examined the resulting estimates of clonal diversity in each patch as the threshold varied, as an independent estimate of the threshold for distinguish within- from among-lineage diversity. (3) We used an assignment test (Meirmans, 2020) to determine whether ramets were appropriately assigned to patches of origin.

We report clonal diversity as the proportion of distinguishable genets (the number of genotypes divided by the total number of individuals or $\mathrm{G} / \mathrm{N}$ ). We examined other aspects of diversity using diversity statistics derived from estimates of population structure provided by the 
R package Hickory v0.1 (https://kholsinger.github.io/Hickory/), which uses Hamiltonian Monte Carlo as implemented in Stan to provide Bayesian estimates of the relevant parameters (including the entire posterior distribution of allele frequencies, within patch inbreeding $\left(F_{\text {IS }}\right)$, and diversity within and among patches with the fixation index $\left.\left(F_{\text {st }}\right)\right)$. Hickory implements an improved version of a Bayesian model first described in Holsinger et al. (2002) and Holsinger and Wallace (2004) that addresses shortcomings noted by Foll et al. (Foll et al., 2008). We use custom R scripts to extract the posterior distribution of allele frequencies and use them to provide estimates of several estimates of diversity.

1. We estimate Nei's Genetic Diversity within each population as $H_{e}^{(p)}=$ $\frac{1}{N_{l}} \sum_{k=1}^{N_{l}}\left(1-p_{k p}^{2}-q_{k p}^{2}\right)$ where $p_{k p}$ and $q_{k p}$ are the frequency of dominant and recessive alleles at locus $k$ in population $p$ and $N_{l}$ is the number of loci. We do not apply the bias correction typically used in estimates of $H_{e}$, because the posterior estimates of allele frequencies already account for sampling uncertainty.

2. We estimate the number of effective alleles per locus (Kimura and Crow, 1964) as $\frac{1}{1-H_{e}}$

3. We estimate the Shannon-Weaver allelic diversity as $H^{(p)}=\frac{1}{N_{l}} \sum_{k=1}^{N_{l}}\left(p_{k p} \ln p_{k p}+\right.$ $\left.q_{k p} \ln q_{k p}\right)$ 
4. Jost (2006) pointed out that a simple transformation of the Shannon-Weaver diversity provides a second index of the effective number of alleles. We estimate the effective number of alleles derived Shannon-Weaver diversity is $\exp \left(H^{(p)}\right)$.

In addition to these estimates of genetic diversity, we also estimated the composite disequilibrium ((Weir, 1996); $\left.\overline{r_{d}}\right)$ because the marker phenotypes cannot be resolved as alleles. Substantial composite disequilibrium is expected as a result of clonal reproduction.

We examined similarity of individuals and genets within and among patches, using both a clustering approach and neighbor-joining trees. To determine the number of clusters to be used, we used an iterative procedure suggested by Grunwald et al. (2016): we iteratively fit a series of principal component analyses (PCA) specifying the use of between 1 and 15 clusters, based on the Prevosti distances. At each of these steps, we sum the within-cluster sums of squares (SS); we chose the number of clusters at which the sum of SS leveled off at an approximate minimum. Within the R package poppr ver. 2.8.2 (Kamvar et al., 2014, 2015), we used the nj() function to make the neighbor-joining trees and the find.clusters function to identify clusters using the principal components data. Finally, to examine whether diversity measurements were related to patch area or patch sample size, we used Pearson's correlations.

We used rarefaction methods to evaluate the effect of the number of markers used to identify the number of MLLs in each patch. To do so we resampled our data into multiple unique 
sets of $10,30,50,70,90,110,130$ and 150 markers and then calculated the mean and variance of the number of MLL detected at each number of markers. We then used graphical analysis to examine the relationship between the number of markers used and the means and variances of the estimated numbers of MLLs.

\section{RESULTS}

We found a total of 186 reproducible loci across 358 individuals using 11 ISSR primers. The number of bands amplified by each primer set ranged from 13 to 23 , and all amplified loci were polymorphic (Table 1). Within each patch, the number of ISSR bands (marker richness) varied from 73 in patch 14 to 134 in patch 8, with differences largely related to sample size (Pearson's $\rho=0.71$ ) associated with differences in patch area. Similarly, the number of polymorphic loci within patches varied considerably, from 29 in patch 14 to 108 in patch 8 (Table 2).

Genotypic and genetic diversity - Every one of the 358 individuals sampled possessed a unique multi-locus genotype (MLG): the number of genets per ramet was 1 based strictly on detected polymorphisms. However, patterns of marker abundance varied considerably among patches. Histograms of the frequency of marker occurrence by patch, show that on the order of 25-50 markers occurred in nearly all individuals within a patch (Fig. 1A). Beyond that, patterns of occurrence varied among patches; in several patches (most notably in patch 8) there was a second mode for markers that were very uncommon in that patch. The heatmap of marker 
presence (Fig. 1B) shows that many markers are quite uncommon (while others are very common) in these samples, and the patches appear to vary in their patterns of relative occurrences of these markers. Overall, we found that many markers occur in over half of the ramets (42 markers, or 23\%, occurred in at least half of individuals; Fig. 2A), while a few markers occur in very few ramets (three markers occurred in only one individual and five occurred in only two). A comparison of the number of differences between pairs of ramets indicates that ramets differ by between 1 and 89 markers with $99.4 \%$ of comparisons differing by more than 10 markers (Fig. 2B).

There is a minimum in the density of pairwise differences at approximately 30 differences (Fig. 2B). This corresponds to a Prevosti distance of 0.064 using the farthest neighbor algorithm (Fig. 3). Using this threshold leads to identification of 13 MLLs across the 358 individuals. Other algorithms (nearest neighbor and UPGMA) gave smaller thresholds that had no correspondence with the approximate minimum in the density plot. Henceforth, we refer to the original $(\mathrm{N}=358)$ data set as the multi-locus genotype (MLG) data set and the reduced (N=13) data set as the MLL data set. This assignment to MLLs indicated that each patch corresponds to a single genetic lineage, with the exception of patch 8 . This patch contains 1 ramet assigned to MLL 2 (otherwise found only in patch 13) and 34 ramets assigned to cluster 13 (found nowhere else). 
Similar results emerged from assignment of individuals to MLLs under an infinite alleles model, using Genodive. The inferred number of MLLs (i.e., richness; Fig 4a) and effective number of MLLs (Fig. 4B) are much smaller than sample sizes for all thresholds from 14 to 28 marker differences for all patches, and they decline rapidly as thresholds are increased for grouping individuals into MLLs. All patches were inferred to belong to a single genetic lineage at greater numbers of marker differences. Patch 8 consistently was inferred to have more MLLs than other patches; for thresholds smaller than 14 the inferred number of MLLs in this patch approached the sample size, so we did not consider smaller thresholds.

Finally, under the assignment test (using $\alpha=0.002$ and 358,000 resampled individuals) no individuals were tagged as migrants.

Using the MLG dataset, the effective number of alleles at each locus is quite low (1.0-1.3) regardless of whether estimates are derived from estimates of Nei's genetic diversity or of Shannon-Weaver allelic diversity (Table 3). Furthermore, estimates of within patch inbreeding range from 0.741 to 0.912 suggesting that genotypes are largely homozygous (Table 3 ). Both observations are consistent with the largely clonal structure of patches and suggest that sexual reproduction, when it occurs, largely involves close relatives or self-fertilization. In contrast, composite disequilibrium $\left(\overline{r_{d}}\right)$ ranges between 0.015 and 0.055 , and is only weakly correlated with sample size ( $\rho=0.12$; Table 3$)$. The resampling tests suggest that the observed composite 
disequilibrium is not likely to reflect sampling error, but the small magnitude of the composite disequilibrium combined with the evidence for strong clonal structure suggests that variation within patches largely reflects mutations that arise independently within clonal lineages.

Genetic structure among patches - Using Hickory, we estimated $F_{\text {st }}$ to be 0.567 with a $95 \%$ credible interval of $(0.519,0.615)$ among patches. Using the Grunwald et al. (2016) approach to choose the optimal number of clusters, we identified 13 statistical clusters that correspond to groups of genotypes more similar to one another than to other genotypes in our sample. These clusters largely corresponded with individual patches. Principal components 1 and 2 of the PCA explained $18 \%$ of the total variation and separated individuals into groups with almost perfect correspondence between patch and statistical cluster (Fig. 5). These two axes alone separate many of the patches completely. Likewise, genetic structure is apparent from the neighborjoining tree, which assigned all L. chinensis ramets into the same thirteen clusters, with almost no mixing among patches (Fig. 6). Examining the PCA assignments, we found that only in patch 8 were ramets assigned to multiple clusters; in every other patch the sampled ramets were assigned to single clusters.

Genetic structure within a patch - Mantel correlograms for the MLG data set found that, in some patches, over short spatial scales, there were clearly positive genetic correlations between individuals. These correlations were significant in five of the 13 patches $(2,3,6,11$ and 14). 
There were no clearly negative correlations. Because only patch 8 had multiple MLLs and it had only two, analysis of MLL structure within a patch would not be informative.

Consequences of number of markers used - Our random resampling approach to subsets of markers indicates (for the MLG data) that the relationship between number of markers used and $\log$ (sample sd of the number of genotypes identified) is strongly linear (Table 4). For $\overline{r_{d}}$, the mean of the estimates approaches its final value around 60 or so markers, but its standard deviation declines exponentially with the number of markers used (Fig. 7).

\section{DISCUSSION}

Successful conservation and restoration efforts depend critically on understanding the genetic make-up of populations, which includes not only genetic diversity, but how that diversity is structured by clonal propagation within populations (Hufford and Mazer, 2003; McKay et al., 2005; Kramer and Havens, 2009). Understanding the genetic make-up of foundation plant species in particular is important for conservation since these species create and define ecological communities and contribute disproportionately to ecosystem function (Ellison et al., 2005; Hughes and Lotterhos, 2014; Lau et al., 2016; Ellison, 2019; Whitham et al., 2020). Foundation grass species often combine clonal and sexual reproduction (Franks et al., 2004;

Keeler et al., 2004; Richards et al., 2004; Tumas et al., 2019). Although our initial analysis found that every ramet of the foundation grass L. chinensis was a unique genotype, further 
consideration of the reproductive biology of the species and the number and type of markers we used, suggest that we have 13 lineages that are closely associated with patches and some small amount of variation that could be due to somatic mutation as well as technical or scoring errors, which requires further study to decipher.

Identifying unique genotypes - Every individual sampled in this study was unique (N=358; G/N $=1.0 ;{ }^{1} D={ }^{2} D=$ sample size for each patch). Several methods of identifying clusters of similar (and presumably closely related) genotypes identified 13 multilocus lineages (MLLs). Within each lineage genotypes are more closely related to each other than they are to genotypes in other lineages. Whether by identifying a minimum in pairwise Prevosti distances or by assigning individuals to lineages using an infinite alleles model, we identified approximately 13 MLLs in this study. Only one of the patches we studied contains more than one MLL identified in this way; G/N is approximately 27.5 for the entire sample, and ${ }^{1} D$ and ${ }^{2} D$ are close to 1 for each patch. For comparison, we used the mean value (0.62) of estimates of the Gini-Simpson index reported by Ellstrand and Roose (1987) for populations of 21 clonal species; this corresponds to an average of ${ }^{2} D=2.63$.

The low number of MLLs found within patches is consistent with extensive clonal spread in L. chinensis (Yang and Li, 1994; Yang et al., 1995; Wang et al., 2005). Furthermore, very few L. chinensis seedlings can be found in the field (Zhu et al., 1981; Yang et al., 1995; Huang et al., 
2002), even if seed production is abundant. A study in the southwestern Heilongjiang Province of China evaluated every ramet (58-79 ramets) of four plots ranging in size from 4.0-4.6 $\mathrm{m}^{2}$, and found only 5-15 genotypes in each patch (G/N from 0.09- 0.21; Hong, 2013).

However, several studies of other clonal species found high clonal diversity. Widén et al. (1994) reported an average of $27 \%$ different unique genotypes in 45 clonal plant species based on isozyme analysis. We suggest that the difficulty may lie, at least partly, in how researchers define clones. Inferring clone membership on the basis of identical genotypes (rather than the basis of being a member of a lineage that was produced from a single sexually produced seed) can lead to confusion, because somatic mutation leads to genetic variation among ramets (Scrosati, 2002; Schoen and Schultz, 2019; Gurevitch et al., 2020). Ellstrand and Roose (1987) reported that the number of genotypes was positively correlated to the number of markers used, and was usually higher in DNA-based than in isozyme-based studies (see also Schoen and Schultz, 2019). Our results from randomly sub-sampling (Fig. 7) our data suggest that, had we used fewer markers, we might have identified many fewer unique genotypes. Studies on another perennial grass Spartina alterniflora reported either that nearly every ramet was unique (Richards et al., 2004; Foust et al., 2016, Robertson et al., 2017) or that the number of ramets per genet varied greatly (Hughes and Lotterhos, 2014). Moreover, Tumas et al. (2019) found that measures of genetic and clonal diversity varied dramatically across the range of the salt marsh 
foundation plant Juncus roemerianus. They found greater genetic diversity and lower clonal diversity in Gulf of Mexico populations than on the Atlantic coast. The authors suggest this could be the result of differences in plant community and disturbance regimes or reflect a relationship with population size. However, none of these studies considered the importance of closely related genets that might actually be more accurately classified as lineages. This type of analysis could inform our understanding of actual functional differences and how they might support adaptive response.

Genetic diversity - Wang et al. (2005) examined 16 natural populations of L. chinensis from different geographical regions and showed that there was significant variation among populations $\left(F_{\mathrm{st}}=0.211, P<0.001\right)$. Yuan et al. (2016) investigated 18 natural populations of $L$. chinensis along a longitudinal gradient from 114 to $124^{\circ} \mathrm{E}$ in northeast China and concluded that genetic diversity was significantly higher in eastern $\left(H_{\exp }=0.628\right)$ than in western sites $\left(H_{\exp }=\right.$ 0.579) $(P<0.01)$. In this study, we found that marker richness among patches ranged from 73134 (out of a total of 186 total markers), and our estimate of $H_{\text {exp }}$ ranged between 0.046 and 0.186 among patches, with an overall 0.308 (Table 2). The population-wide estimate from Hickory shows a large degree of differentiation between patches $\left(F_{\mathrm{st}}=0.567 ; 95 \%\right.$ credible interval $=((0.519,0.615))$. By contrast the pooled estimate of $H_{\text {exp }}$ is $0.347(0.333,0.362)$. Our among-patch differentiation thus appears to be considerably larger than in previously studied 
populations of L. chinensis, and our within-patch variation lower. The percent polymorphic loci (40-81\%) among patches is on the same order of magnitude as reported for outcrossing grasses (50-60\% for regional or widespread outcrossers; Hamrick and Godt, 1996; Godt and Hamrick, 1998).

Given that clonal growth is extensive and previous studies have found less than $1 \%$ survival rate of seedlings in L. chinensis natural populations (Benson et al., 2004; Dalgleish and Hartnett, 2006), it is likely that the high level of genetic variation could be due to somatic mutation, or occasional cross pollination, and long life span. Somatic mutation would create unique loci and contribute to the high diversity observed within patches of L. chinensis (Antolin and Strobeck, 1985). Lynch (1984) and Gill et al. (1995) both reported that high rates of somatic mutation could compensate for the absence of recombination from sexual reproduction in primarily asexual species, and may allow asexual species to maintain abundant genetic variation and adapt to changing environmental conditions. However, the mutation rate in plants in general is poorly understood (Schoen and Schultz, 2019). This is also true among clonal plant species, despite the fact that several authors have argued the importance of somatic mutation in clonal plants (Eckert, 2002; Fischer and van Kleunen, 2002). Somatic mutation has generally been considered disadvantageous with the expectation that there should be selection for low somatic mutation rates. On the contrary, in a recent review of the impacts of somatic mutations in evolution 
Schoen and Schultz (2019) argue that in plants, "somatic mutation might be relatively innocuous or even beneficial, as the indeterminate and partially independent modular design of most plants may allow cell lines to be lost without sacrificing overall plant function and can amplify the occurrence of beneficial mutations." They also present evidence that the contributions of somatic mutation to population genetic variability varies in different species. For instance, high genotypic diversity in Australian populations of Grevillea rhizomatosa (Proteaceae) was attributed to somatic mutations and $25 \%$ of connected ramets had differences based on ISSR markers (Gross et al., 2012). Similarly, in New Zealand populations of Hieracium pilosella, high diversity of ISSR genotypes could have resulted from somatic mutation (Houliston and Chapman, 2004). In our study, somatic mutation might be indicated by markers that are only found in a few individuals or if pairwise comparisons indicated that many ramets were differentiated by only a few markers. However, we found that only eight markers $(2 \%)$ were found in only one or two ramets. Moreover, more than $99 \%$ of ramets differed by more than 10 markers. Then again, these expectations might not be reasonable depending on the age of the patches and the accumulation of multiple mutations within MLLs. Further work is required to identify the importance of somatic mutation in these populations.

Although the capacity for sexual reproduction of L. chinensis is reportedly rather weak, frequent somatic mutations and some level of sexual reproduction could generate considerable 
variation. These loci would be accumulated through extensive clonal propagation and thus, each patch would exhibit a high level of genetic diversity. We evaluated the number of markers that would be effective at identifying the minimum number of MLL in this study to be 60 or so markers instead of the 186 that we used here. We suggest further study will be required to evaluate how many markers to use, and how to interpret the resulting data, given the possible maintenance of somatic mutations in highly clonal plants. The contribution of somatic mutation could be an important consideration for markers like microsatellites that already have high expected mutation rates.

Genetic structure among patches - Clear and strong genetic structure among the thirteen patches were observed both in PCA plots and neighbor-joining trees providing evidence for genetic isolation among these patches, and a lack of gene flow among patches. Gene flow can occur at several life stages in plant species: as pollen, fertilized seeds or rhizome movement via water or animal vectors or disturbance. Previous studies report low pollen viability, short pollen longevity and short pistil receptivity of L. chinensis lead to low seed production (Huang et al., 2004). The L. chinensis seeds do not have wings or plumes for dispersal by wind. Thus, lack of dispersal ability could result in a decreased possibility of sexually produced genetic exchange. In addition, 
isolation could result from the history of colonization of a patch by specific genotypes with subsequent accumulation of mutations.

If somatic mutation is an important source of variation, the differences among patches could reflect an age-based structure as mutations accumulate over time. However, a study of the full genome of a single 234-year-old European oak (Quercus robur), found 17 point mutations while another study found almost three times as many point mutations in an even younger tree (Schmid-Siegert et al., 2017; Plomion et al., 2018). Up to seven out of 19 mutations were also transmitted to offspring indicating inheritance of these differences not only through vegetative reproduction but through sexual (Plomion et al., 2018). In a study of aspen, Ally et al. (2010) found that the number of somatic mutations at 14 microsatellite loci was a good proxy for individual plant age, while plant (clone) size alone was not a reliable indicator.

Finally, there may be spatial variation of selection intensity induced by environmental heterogeneity (Linhart and Grant, 1996). Owing to microhabitat heterogeneity, alleles within plant populations can have a strong spatial structure (Hamrick and Holden, 1979). If this process is partly responsible for the variation among patches, it would be operating because of linkage between ISSR loci and loci under selection. Thus inferences about it would require much more information about the Leymus genome as well as about spatially heterogenous selection. Identifying the contribution of limited migration, somatic mutation, age and microhabitat 
heterogeneity to the genetic structure among patches of L. chinensis will require more detailed studies of these populations and environmental factors.

Genetic structure within a patch - We detected significant and positive correlations between geographic and genetic distances among individuals within either the three smallest patches (11, $13,14)$ or the four largest patches $(2,3,6,8)$ with Mantel tests, indicating that geographic distance is a factor shaping the genetic structure of these patches. Yang and Zhang (2006) reported that with increasing patch area, the number of total ramets increased linearly in $L$. chinensis patches. The clonal architecture of L. chinensis tended to be spatially clustered in patches 13 and 14. In this case, neighboring ramets are expected to be closely related, such that positive autocorrelation may exist within a short distance. Clonal growth form can greatly influence the distribution of genetic variation within clonal patch.

For the largest patches (2, 3, 6 and 8), low ramet densities and larger spatial distances between ramets, may have further restricted gene flow among individuals. The observed negative correlations over the longest distance in patches 6 and 8 indicated genetic differentiation within a patch may not occur in patches smaller than 8 meters in diameter. In summary, patch size plays an important role in shaping the genetic structure among individuals within patches.

Implications for conservation - Genetic diversity is the material basis for the existence and development of populations, and protecting genetic diversity of populations is an important task. 
Several authors acknowledge that while foundation species are common and abundant, and therefore often not considered for conservation purposes, protecting these species is critical for maintaining ecosystem function (Ellison, 2019; Qiao et al., 2021). Leymus chinensis is a candidate foundation species in the Inner Mongolia steppe region, where overgrazing has led to severe degradation (Bai et al., 2004; Tong et al., 2004). In addition, these grasslands face extreme climate events and chronic environmental challenges that are partly buffered by this species (Bai et al., 2010; Meng et al., 2019). Restoration of these degraded populations could depend on management of patchy populations. In order to do so, it is necessary to understand how genetic diversity is structured within and among patches before formulating a conservation or restoration strategy, bearing in mind that a recent study reported significant variation among 10 L. chinensis genotypes in response to drought, high temperature, and both drought and high temperature (Yang et al., 2019). Our results indicated larger patches have higher levels of genetic diversity, but patches themselves are frequently derived from a single lineage. These findings provide important reference information for management agencies. Although we do not know how functionally different these MLLs are, we suggest that the conservation management of these patchy populations should keep to the following principles: if conditions permit, it is better to protect as many patches as possible; if resources are limited, local managers should give priority to protecting several large patches because of their high genetic diversity, and increased 
potential for functional diversity. Maximizing the retention of genetic diversity will be important for the long-term survival of patchy populations.

\section{ACKNOWLEDGMENTS}

The authors thank Qian Xiong for assistance with the experiment. The study was financially supported by National Science Foundation of China (Grant No. 32071860 and 31570332 ),

Natural Science Foundation of Science and Technology Department of Liaoning Province (Grant No. 2019-MS-155), Serving Local Project of Education Department of Liaoning Province (Grant No. LFW202001), and Federal Ministry of Education and Research (BMBF; MOPGA Project ID 306055 to CLR). The authors gratefully acknowledge financial support from China Scholarship Council.

\section{AUTHOR CONTRIBUTIONS}

The project was designed and organized by Chan Zhou. Jian Guo and Zhuo Zhang conducted and managed data collection. Gordon A. Fox coordinated statistical analysis. Zhuo Zhang provided critical molecular expertise throughout the project. Kent E. Holsinger and Christina L. Richards provided expertise in population genetics. All authors contributed to the writing of the manuscript. 


\section{DATA AVAILABILITY}

Data and code for data analysis will be archived with Datadryad (http://datadryad.org) upon acceptance of the paper.

\section{LITERATURE CITED}

Ainouche, M. L., A. Baumel, and A. Salmon. 2004. Spartina anglica C. E. Hubbard: a natural model system for analysing early evolutionary changes that affect allopolyploid genomes. Biological Journal of the Linnean Society 82: 475-484.

Ainouche, M. L., P. Fortune, A. Salmon, C. Parisod, M.-A. Grandbastien, K. Fukunaga, and M.T. Misset. 2009. Hybridization, polyploidy and invasion: lessons from Spartina (Poaceae). Biological Invasions 11: 1159-1173.

Ally, D., K. Ritland, and S. P. Otto. 2010. Aging in a long-lived clonal tree. PLOS Biology 8: e1000454.

Antolin, M., and C. Strobeck. 1985. The population genetics of somatic mutation in plants. American Naturalist 126: 52-62.

Arnaud-Haond, S., C. M. Duarte, F. Alberto, and E. A. Serrão. 2007. Standardizing methods to address clonality in population studies. Molecular Ecology 16: 5115-5139. 
Bai, Y., X. Han, J. Wu, Z. Chen, and L. Li. 2004. Ecosystem stability and compensatory effects in the Inner Mongolia grassland. Nature 431: 181-184.

Bai, W. M., X. Q. Sun, Z. W. Wang, and L. H. Li. 2009. Nitrogen addition and rhizome severing modify clonal growth and reproductive modes of Leymus chinensis population. Plant Ecology 205: 13-21.

Bai, Y., J. Wu, C. M. Clark, S. Naeem, Q. Pan, J. Huang, L. Zhang, and X. Han. 2010. Tradeoffs and thresholds in the effects of nitrogen addition on biodiversity and ecosystem functioning: evidence from inner Mongolia Grasslands. Global change biology 16: 358-372.

Benson, E. J., D. C. Hartnett, and K. H. Mann. 2004. Below-ground bud banks and meristem limitation in tallgrass prairie plant populations. American Journal of Botany 91: 416-421.

Chen, J. M., W. R. Gituru, Y. H. Wang, and Q. F. Wang. 2006. The extent of clonality and genetic diversity in the rare Caldesia grandis (Alismataceae): comparative results for RAPD and ISSR markers. Aquatic Botany 84: 301-307.

Chen, S., J. Jia, L. Chen, P. Zhao, D. Qi, W. Yang, H. Liu, et al. 2019. Transcriptomic analysis reveals a comprehensive calcium- and phytohormone-dominated signaling response in Leymus chinensis self-incompatibility. International Journal of Molecular Sciences 20: 2356. 
Daehler, C. C., and D. R. Strong. 1994. Variable reproductive output among clones of Spartina alterniflora (Poaceae) invading San-Francisco Bay, California: the influence of herbivory, pollination, and establishment site. American Journal of Botany 81: 307-313.

Dalgleish, H. J., and D. C. Hartnett. 2006. Below-ground bud banks increase along a precipitation gradient of the North American Great Plans: a test of the meristem limitation hypothesis. New Phytologist 171: 81-89.

Davis, H. G., C. M. Taylor, J. C. Civille, and D. R. Strong. 2004. An Allee effect at the front of a plant invasion: Spartina in a Pacific estuary. Journal of Ecology 92: 321-327.

Douhovnikoff, V., and R. S. Dodd. 2003. Intra-clonal variation and a similarity threshold for identification of clones: application to Salix exigua using AFLP molecular markers. Theoretical and Applied Genetics 106: 1307-1315.

Doyle, J. J., and J. L. Doyle. 1987. A rapid DNA isolation procedure for small quantities of fresh leaf tissue. Phytochemical Bulletin 19: 11-15.

Eckert, C. G. 2002. The loss of sex in clonal plants. Evolutionary Ecology 15: 501-520.

Ellison, A. M. 2019. Foundation species, non-trophic interactions and the value of being common. iScience 13: 254-268. 
Ellison, A. M., M. S. Bank, B. D. Clinton, E. A. Colburn, K. Elliott, C. R. Ford, D. R. Forster, et al. 2005. Loss of foundation species: consequences for the structure and dynamics of forested ecosystems. Frontiers in Ecology and the Environment 3: 479-486.

Ellstrand, N. C., and M. L. Roose. 1987. Patterns of genotypic diversity in clonal plant species. American Journal of Botany 74: 123-131.

Fischer, M., and M. van Kleunen. 2002. On the evolution of clonal plant life histories. Evolutionary Ecology 15: 565-582.

Foll, M., M. A. Beaumont, and O. Gaggiotti. 2008. An approximate Bayesian computation approach to overcome biases that arise when using amplified fragment length polymorphism markers to study population structure. Genetics 179: 927-939.

Foust, C. M., V. Preite, A. W. Schrey, M. Alvarez, M. H. Robertson, K. J. F. Verhoeven, and C. L. Richards. 2016. Genetic and epigenetic differences associated with environmental gradients in replicate populations of two salt marsh perennials. Molecular Ecology 25: $1639-1652$.

Frankel, O. H. 1970. Genetic dangers in the green revolution. World Agriculture 19: 9-13.

Frankel, O. H., and M. E. Soulé. 1981. Conservation and evolution. Cambridge University Press, Cambridge, UK. 
Franks, S. J., C. L. Richards, E. Gonzales, J. E. Cousins, and J. L. Hamrick. 2004. Multi-scale genetic analysis of Uniola paniculata (Poaceae): a coastal species with a linear, fragmented distribution. American Journal of Botany 91: 1345-1351.

Gao, L., Y. Geng, B. Li, J. Chen, and J. Yang. 2010. Genome-wide DNA methylation alterations of Alternanthera philoxeroides in natural and manipulated habitats: implications for epigenetic regulation of rapid responses to environmental fluctuation and phenotypic variation. Plant, Cell \& Environment 33: 1820-1827.

Gardner, S. N., and M. Mangel. 1999. Modeling investments in seed, clonal offspring, and translocation in a clonal plant. Ecology 80: 1202-1220.

Gill, D. E., L. Chao, S. L. Perkins, and J. B. Wolf. 1995. Genetic mosaicism in plants and clonal animals. Annual Review of Ecology and Systematics 26: 423-444.

Godt, M. J. W., and J. L. Hamrick. 1998. Allozyme diversity in the grasses. In G. P. Cheplick (ed.), Population biology of the grasses, 11-29. Cambridge University Press, Cambridge, UK.

Gray, M. M., P. St. Amand, N. M. Bello, M. B. Galliart, M. Knapp, K. A. Garrett, T. J. Morgan, et al. 2014. Ecotypes of an ecologically dominant prairie grass (Andropogon gerardii) exhibit genetic divergence across the U.S. Midwest grasslands' environmental gradient. Molecular Ecology 23: 6011-6028. 
Gross, C. L., P. A. Nelson, A. Haddadchi, and M. Fatemi. 2012. Somatic mutations contribute to genotypic diversity in sterile and fertile populations of the threatened shrub, Grevillea rhizomatosa (Proteaceae). Annals of Botany 109: 331-342.

Grunwald, N., Z. N. Kamvar, and S. E. Everhart. 2016. grunwaldlab/Population_Genetics_in_R: First release [Data set]. Zenodo. https://doi.org/10.5281/zenodo.160588. [accessed 10 January 2020].

Gurevitch, J., S. M. Scheiner, and G. A. Fox. 2020. The ecology of plants. 3rd ed. Sinauer Associates, Sunderland, MA.

Guo, J., H. Li, and Y. Yang. 2020. Phenotypic plasticity in sexual reproduction based on nutrients supplied from vegetative ramets in a Leymus chinensis population. Frontiers in Plant Science 10: 1681.

Hamrick, J. L., and M. J. W. Godt. 1996. Effects of life history traits on genetic diversity in plant species. Philosophical Transactions of the Royal Society London, B, Biological Sciences 351: $1291-1298$.

Hamrick, J. L., and L. R. Holden. 1979. Influence of microhabitat heterogeneity on gene frequency distribution and gametic phase disequilibrium in Avena barbata. Evolution 33: 521. 
Han, Y. C., C. Z. Teng, S. Zhong, M. Q. Zhou, Z. L. Hu, and Y. C. Song. 2007. Genetic variation and clonal diversity in populations of Nelumbo nucifera (Nelumbonaceae) in central China detected by ISSR markers. Aquatic Botany 86: 69-75.

Holsinger, K. E. 2000. Reproductive systems and evolution in vascular plants. Proceedings of the National Academy of Sciences of the United States of America 97: 7037-7042.

Holsinger, K. E., P. O. Lewis, and D. K. Dey. 2002. A Bayesian approach to inferring population structure from dominant markers. Molecular ecology 11: 1157-1164.

Holsinger, K. E., and L. E. Wallace. 2004. Bayesian approaches for the analysis of population genetic structure: an example from Platanthera leucophaea (Orchidaceae). Molecular ecology 13: 887-894.

Hong, R. M. 2013. Genetic dissimilarity of clonal populations of Leymus chinensis (Trin.) Tzvel in heterogeneous and homogeneous habitats in southwestern Heilongjiang Province of China. Journal of Northeast Agricultural University (English Edition) 20: 21-29.

Houliston, G. J., and H. M. Chapman. 2004. Reproductive strategy and population variability in the facultative apomict Hieracium pilosella (Asteraceae). American Journal of Botany 91: $37-44$. 
Huang, Z., J. Zhu, X. Mu, and J. Lin. 2004. Pollen dispersion, pollen viability and pistil receptivity in Leymus chinensis. Annals of Botany 93: 295-301.

Huang, Z. H., J. M. Zhu, X. J. Mu, and J. X. Lin. 2002. Advances on the mechanism of low sexual reproductivity of Leymus Chinensis. Grassland of China 24: 55-60.

Hufford, K. M., and S. J. Mazer. 2003. Plant ecotypes: genetic differentiation in the age of ecological restoration. Trends in Ecology and Evolution18: 147-155.

Hughes, A. R., B. D. Inouye, M. T. J. Johnson, N. Underwood, and M. Vellend. 2008. Ecological consequences of genetic diversity. Ecology Letters 11: 609-623.

Hughes, A. R., and K. E. Lotterhos. 2014. Genotypic diversity at multiple spatial scales in the foundation marsh species, Spartina alterniflora. Marine Ecology Progress Series 497: 105117.

Inner-Mongolia and Ning Xia Investigation and Survey Team of the Chinese Academy of Sciences. 1985. The vegetation of Inner Mongolia. Science Press, Beijing, China pp 516527.

Jost, L. 2006. Entropy and diversity. Oikos 113: 363-375.

Kamvar, Z. N., J. C. Brooks, and N. J. Grünwald. 2015. Novel R tools for analysis of genomewide population genetic data with emphasis on clonality. Frontiers in Genetics 6: 208. 
Kamvar, Z. N., J. F. Tabima, and N. J. Grünwald. 2014. Poppr: an R package for genetic analysis of populations with clonal, partially clonal, and/or sexual reproduction. PeerJ 2: e281.

Keeler, K. H. 2004. Impact of intraspecific polyploidy in Andropogon gerardii (Poaceae) populations. American Midland Naturalist 152: 63-74.

Keeler, K. H., C. F. Williams, and L. S. Vescio. 2002. Clone size of Andropogon gerardii vitman (big bluestem) at Konza Prairie, Kansas. American Midland Naturalist 147: 295-304.

Keith, A. R., J. K. Bailey, M. K. Lau, and T. G. Whitham. 2017. Genetics-based interactions of foundation species affect community diversity, stability and network structure. Proceedings. Biological sciences 284: 20162703.

Kimura, M., and J. F. Crow. 1964. The number of alleles that can be maintained in a finite population. Genetics 49: 725-738.

Klimeš, L., J. Klimešova, R. Hendriks, and J. van Groenendael. 1997. Clonal plant architecture: a comparative analysis of form and function. In H. de Kroon \& J. M. van Groenendael [eds.], The ecology and evolution of clonal plants, pp. 1-29. Backhuys Publishers, Leiden, Netherlands.

Kramer, A. T., and K. Havens. 2009. Plant conservation genetics in a changing world. Trends in Plant Sciences 14: 599-607. 
Lehmann, C. 1997. Clonal diversity of populations of Calamagrostis epigejos in relation to environmental stress and habitat heterogeneity. Ecography 20: 483-490.

Lau, M. K., A. R. Keith, S. R. Borrett, S. M. Shuster, and T. G. Whitham. 2016. Genotypic variation in foundation species generates network structure that may drive community dynamics and evolution. Ecology 97: 733-742.

Li, Y.Y., X.-T. Lü, Z.-W. Wang, C. Zhou, and X.-G. Han. 2014. Linking relative growth rates to biomass allocation: the responses of the grass Leymus chinensis to nitrogen addition. PhytonInternational Journal of Experimental Botany 83: 283-289.

Linhart, Y. B., and M. C. Grant. 1996. Evolutionary significance of local genetic differentiation in plants. Annual Review of Ecology and Systematics 27: 237-277.

Liu, S. H., Y. H. Kang, S. Q. Wan, Z. C. Wang, Z. W. Liang, and X. J. Sun. 2011. Water and salt regulation and its effects on Leymus chinensis growth under drip irrigation in saline-sodic soils of the Songnen Plain. Agricultural Water Management 98: 1469-1476.

Lynch, M. 1984. Destabilizing hybridization, general-purpose genotypes and geographic parthenogenesis. Quarterly Review of Biology 59: 257-290.

Lynch, M., R. Burger, D. Butcher, and W. Gabriel. 1993. The mutational meltdown in asexual populations. Journal of Heredity 84: 339-344. 
McKay, J. K., C. E. Christian, S. Harrison, and K. J. Rice. 2005. “How local is local?” A review of practical and conceptual issues in the genetics of restoration. Restoration Ecology 13: $432-440$.

Meirmans, P. G. 2020. Genodive version 3.0: easy-to-use software for the analysis of genetic data of diploids and polyploids. Molecular Ecology Resources 20: 1126-1131.

Meirmans, P. G., and P. H. Van Tienderen. 2004. Genotype and genodive: two programs for the analysis of genetic diversity of asexual organisms. Molecular Ecology Notes 4: 792-794.

Meng, B., B. Shi, S. Zhong, H. Chai, S. Li, Y. Wang, H. A. L. Henry, et al. 2019. Drought sensitivity of aboveground productivity in Leymus chinensis meadow steppe depends on drought timing. Oecologia 191: 685-696.

Morris, G. P., P. P. Grabowski, and J. O. Borovitz. 2011. Genomic diversity in switchgrass (Panicum virgatum): from the continental scale to a dune landscape. Molecular Ecology 20: $4938-4952$.

Mounger, J., M. L. Ainouche, O. Bossdorf, A. Cavé-Radet, B. Li, M. Parepa, A. Salmon, et al. 2021. Epigenetics and the success of invasive species. Philosophical Transactions of the Royal Society B 376: 20200117. 
Plomion, C., J. M. Aury, J. Amselem, T. Leroy, F. Murat F, S. Duplessis, S. Faye, et al. 2018.

Oak genome reveals facets of long lifespan. Nature Plants 4: 440-452.

Qiao, X., J. Zhang, Z. Wang, Y. Xu, T. Zhou, X. Mi, M. Cao, et al. 2021. Foundation species across a latitudinal gradient in China. Ecology 102: e03234.

R Core Team. 2017. R: a language and environment for statistical computing. R Foundation for Statistical Computing, Vienna, Austria.

Raabová, J., F. V. Rossum, A. L. Jacquemart, and O. Raspé. 2015. Population size affects genetic diversity and fine-scale spatial genetic structure in the clonal distylous herb Menyanthes trifoliata. Perspectives in Plant Ecology 17: 193-200.

Reynolds, L. K., K. J. McGlathery, and A. M. Waycott. 2012. Genetic diversity enhances restoration success by augmenting ecosystem services. PLoS One 7: e38397.

Richards, A. J. 1986. Plant breeding systems. George Allen \& Unwin, London, UK.

Richards, C. L., J. L. Hamrick, L. A. Donovan, and R. Mauricio. 2004. Unexpectedly high clonal diversity of two salt marsh perennials across a severe environmental gradient. Ecology Letters 7: 1155-1162. 
Richards, C. L., A. W. Schrey, and M. Pigliucci. 2012. Invasion of diverse habitats by few Japanese knotweed genotypes is correlated with epigenetic differentiation. Ecology Letters 15: 1016-1025.

Robertson, M., A. Schrey, A. Shayter, C. J. Moss, and C. L. Richards. 2017. Genetic and epigenetic variation in Spartina alterniflora following the Deepwater Horizon oil spill. Evolutionary Applications 10: 792-801.

Sakai, S. 1995. Optimal resource allocation to vegetative and sexual reproduction of a plant growing in a spatially varying environment. Journal of Theoretical Biology 175: 271-282.

Schaal, B. A., W. J. Leverich, and S. H. Rogstad. 1991. Genetics and conservation of rare plants. Oxford University Press, New York, NY, USA.

Schmid-Siegert, E., N. Sarkar, C. Iseli, S. Calderon, C. Gouhier-Darimont, J. Chrast, P. Cattaneo, et al. 2017. Low number of fixed somatic mutations in a long-lived oak tree. Nature Plants 3: $926-929$.

Schoen, D. J., and S. T. Schultz. 2019. Somatic mutation and evolution in plants. Annual Review of Ecology, Evolution, and Systematics 50: 49-73.

Scrosati, R. 2002. An updated definition of genet applicable to clonal seaweeds, bryophytes, and vascular plants. Basic \& Applied Ecology 3: 97-99. 
Shimizu, Y., M. Ando, and F. Sakai. 2002. Clonal structure of natural populations of Cryptomeria japonica growing at different positions on slopes, detected using RAPD markers. Biochemical Systematics and Ecology 30: 733-748.

Slatkin, M. 1987. Gene flow and the geographic structure of natural populations. Science 236: $787-792$.

Sole, M., W. Durka, S. Eber, and R. Brandl. 2004. Genotypic and genetic diversity of the common weed Cirsium arvense (Asteraceae). International Journal of Plant Science 165: 437-444.

Sork, V. L., S. N. Aitken, R. J. Dyer, A. J. Eckert, P. Legendre, and D. B. Neale. 2013. Putting the landscape into the genomics of trees: approaches for understanding local adaptation and population responses to changing climate. Tree Genetics \& Genomes 9: 901-911.

Tong, C., J. Wu, S. Yong, J. Yang, and W. Yong. 2004. A landscape-scale assessment of steppe degradation in the Xilin River Basin, Inner Mongolia, China. Journal of Arid Environments 59: $133-149$.

Travis, S., and M. Hester. 2005. A space-for-time substitution reveals the long-term decline in genotypic diversity of a widespread salt marsh plant, Spartina alterniflora, over a span of 1500 years. Journal of Ecology 93: 417-430. 
Tumas, H. R., B. M. Shamblin, M. S. Woodrey, and C. J. Nairn. 2019. Broad-scale patterns of genetic diversity and structure in a foundational salt marsh species black needlerush (Juncus roemerianus). Conservation Genetics 20: 903-915.

Van Groenendael, J., and H. de Kroon. 1990. Regulation and function of clonal growth in plants: an evaluation. In J. M. van Groendendal and H. de Kroon [eds.], Clonal growth in plants: regulation and function, pp. 177-186. SPB Academic Publishing, The Hague, Netherlands.

Verhoeven, K. J. F., and V. Preite. 2014. Epigenetic variation in asexually reproducing organisms. Evolution 68: 644-655.

Wang, R. 2001. Comparative study on clonal and sexual reproductive traits of Leymus chinensis populations in different habitats. Journal of Applied Ecology 12: 379-383.

Wang, Y. S., L. M. Zhao, H. Wang, J. Wang, D. M. Huang, R. M. Hong, X. H. Teng, and N. Miki. 2005. Molecular genetic variation in a clonal plant population of Leymus chinensis (Trin.) Tzvel. Journal of Integrative Plant Biology 47: 1055-1064.

Weir, B. S. 1996. Genetic data analysis II: methods for discrete population genetic data. Sinauer.

Whitham, T. G., J. K. Bailey, J. A. Schweitzer, S. M. Shuster, R. K. Bangert, C. J. LeRoy, E. V. Lonsdorf, et al. 2006. A framework for community and ecosystem genetics: from genes to ecosystems. Nature Reviews Genetics, 7: 510-523. 
Whitham, T. G., G. J. Allan, J. A. Schweitzer, H. F. Cooper, and S. M. Shuster. 2020.

Intraspecific genetic variation and species interactions contribute to community evolution. Annual Review of Ecology, Evolution, and Systematics 51: 587-612.

Widén, B., N. Cronberg, and M. Widén. 1994. Genotypic diversity, molecular markers and spatial distribution of genets in clonal plants, a literature survey. Folia Geobotanica 29: $245-263$.

Xu, Z. Z., and G. S. Zhou. 2007. Photosynthetic recovery of a perennial grass Leymus chinensis after different periods of soil drought. Plant Production Science 10: 277-285.

Yang, Y., and J. Li. 1994. Effects of different utilization methods on reproductive characters of Leymus chinensis. Grassland of China 5: 34-37.

Yang, Y., G. Liu, and B. Zhang. 1995. An analysis of age structure and the strategy for asexual propagation of Leymus chinensis population. Acta Botanica Sinica 37: 147-153.

Yang, Y., and B. Zhang. 2006. Growth patterns of Leymus chinensis clones under different habitat conditions in Songnen Plain of China. Journal of Applied Ecology 17: 1419-1423.

Yang, X., J. Li, T. Zhao, L. Mo, J. Zhang, H. Ren, N. Zhao, and Y. Gao. 2019. Variation and heritability of morphological and physiological traits among Leymus chinensis genotypes under different environmental conditions. Journal of Arid Land 11: 66-74. 
Yuan, S., L. Ma, C. Guo, and R. Wang. 2016. What drives phenotypic divergence in Leymus chinensis (Poaceae) on large-scale gradient, climate or genetic differentiation? Scientific Reports 6: 26288.

Zhang, W. D., S. Y. Chen, G. S. Liu, and C. C. Jan. 2004. Seed-set and pollen-stigma compatibility in Leymus chinensis. Grass and Forage Science 59: 180-185.

Zhang, W. D., G. J. Dong, Q. Y. Shu, H. J. Li, and G. S. Liu. 2005. Effect of storage conditions on seed germination, seedling growth and genetic stability in Chinese leymus (Leymus chinensis). Seed Science and Technology 33: 431-440.

Zhang, Y.-Y., M. Parepa, M. Fischer, and O. Bossdorf. 2016. Epigenetics of colonizing species? A study of Japanese knotweed in Central Europe. In S. Barrett, R. I. Colautti, K. M. Dlugosch, and L. H. Rieseberg [eds.], Invasion genetics: the baker and Stebbins legacy, 328-340. Wiley-Blackwell UK.

Zhou, C., Z. Wang, J. Guo, Z. Zhang, and Y. Yang. 2015. Clonal patch size and ramet position of Leymus chinensis affected reproductive allocation. PLoS One 10: e0140350.

Zhou, C., Z. Zhang, Z. Wang, and Y. Yang. 2014. Difference in capacity of physiological integration between two ecotypes of Leymus chinensis underlies their different performance. Plant Soil 383: 191-202. 
Zhou, Z. H., M. Miwa, K. Nara, B. Y. Wu, H. Nakaya, C. L. Lian, N. Miyashita, et al. 2003.

Patch establishment and development of a clonal plant, Polygonum cuspidatum, on Mount Fuji. Molecular Ecology 12: 1361-1373.

Zhu, T. C. 2004. Biological ecology of Leymus chinensis. Jilin Science and Technology Press, Changchun, China.

Zhu, T. C., J. D. Li, and D. C. Yang. 1981. A study of the ecology of yang-cao (Leymus chinensis) grassland in northern China. In J. A. Smith and V. W. Hays [eds.], Proceedings of the XIV international grassland congress, 429-431. Lexington, Kentucky, USA. 


\section{TABLES}

TABLE 1. Basic properties of the 11 ISSR primers producing 186 polymorphic loci: annealing temperature in PCR $\left(\mathrm{T}_{\mathrm{A}}\right)$, number of bands scored $(\mathrm{N}-$ bands): $100 \%$ of scored bands were polymorphic.

\begin{tabular}{|c|c|c|c|}
\hline Primer & Primer sequence (5'-3') & $\mathbf{T}_{\mathbf{A}}\left({ }^{\circ} \mathbf{C}\right)$ & N-bands \\
\hline 6 & $\mathrm{HVH}(\mathrm{CA})_{7} \mathrm{~T}$ & 58 & 19 \\
\hline 10 & $(\mathrm{CA})_{8} \mathrm{RG}$ & 58 & 14 \\
\hline 13 & $(\mathrm{AC})_{8} \mathrm{YG}$ & 56 & 16 \\
\hline 807 & $(\mathrm{AG})_{8} \mathrm{~T}$ & 54 & 13 \\
\hline 811 & $(\mathrm{GA})_{8} \mathrm{C}$ & 56 & 23 \\
\hline 818 & $(\mathrm{CA})_{8} \mathrm{G}$ & 58 & 15 \\
\hline 827 & $(\mathrm{AC}){ }_{8} \mathrm{G}$ & 56 & 18 \\
\hline 834 & $(\mathrm{AG})_{8} \mathrm{YT}$ & 54 & 14 \\
\hline 842 & $(\mathrm{GA})_{8} \mathrm{YG}$ & 55 & 15 \\
\hline 848 & $(\mathrm{CA})_{8} \mathrm{RG}$ & 58 & 19 \\
\hline 855 & $(\mathrm{AC})_{8} \mathrm{YT}$ & 57 & 20 \\
\hline
\end{tabular}


TABLE 2. Patch area, numbers of ramets sampled, ISSR markers found (richness), number of polymorphic loci, and percent polymorphic loci (PL) by patch.

\begin{tabular}{|c|c|c|c|c|c|}
\hline Patch ID & $\operatorname{Area}\left(\mathbf{m}^{2}\right)$ & $\mathrm{N}$ ramets & Marker richness & N polymorphic loci & PL (\%) \\
\hline 1 & 13.98 & 26 & 98 & 56 & 57 \\
\hline 2 & 57.70 & 33 & 100 & 62 & 62 \\
\hline 3 & 69.81 & 35 & 103 & 75 & 73 \\
\hline 5 & 8.40 & 26 & 93 & 66 & 71 \\
\hline 6 & 97.23 & 34 & 112 & 85 & 76 \\
\hline 7 & 8.64 & 25 & 78 & 44 & 56 \\
\hline 8 & 166.79 & 35 & 134 & 108 & 81 \\
\hline 9 & 35.57 & 29 & 75 & 45 & 60 \\
\hline 10 & 22.11 & 27 & 87 & 62 & 71 \\
\hline 11 & 13.77 & 26 & 103 & 72 & 70 \\
\hline 12 & 19.29 & 27 & 109 & 84 & 77 \\
\hline 13 & 3.24 & 18 & 80 & 33 & 41 \\
\hline 14 & 2.78 & 17 & 73 & 29 & 40 \\
\hline Total & & 358 & 186 & 186 & 100 \\
\hline
\end{tabular}


TABLE 3. Estimates of genetic diversity. $N$ (number of samples, equal to the number of genotypes in the MLG dataset), allelic diversity $\left(H_{e}\right.$ : expected heterozygosity, $n_{e}$ : effective number of alleles), Shannon-Weaver diversity $(H$ : entropy, $\exp (H)$ : effective number of alleles) and Inbreeding coefficients $\left(F_{I S}\right)$. Allelic diversity, Shannon-Weaver diversity and inbreeding coefficients are reported as posterior means and $95 \%$ credible intervals based on allele frequency estimates from Hickory. $r_{d}$ is an estimate of average composite disequilibrium in each population, and $P\left(r_{d}\right)$ is a test of the null hypothesis that $r_{d}=0$, based on a resampling approach.

\begin{tabular}{|c|c|c|c|c|c|c|c|c|}
\hline \multirow[b]{2}{*}{ Patch } & \multirow[b]{2}{*}{$\mathbf{N}$} & \multicolumn{2}{|c|}{ Allelic diversity } & \multicolumn{2}{|c|}{$\begin{array}{c}\text { Shannon-Weaver } \\
\text { diversity }\end{array}$} & Inbreeding & \multicolumn{2}{|c|}{$\begin{array}{c}\text { Composite } \\
\text { disequilibrium }\end{array}$} \\
\hline & & $H_{e}$ & $n_{e}$ & $H$ & $\exp (H)$ & $F_{I S}$ & $r_{d}$ & $\mathrm{P}\left(r_{d}\right)$ \\
\hline \multirow[t]{2}{*}{1} & 26 & 0.111 & 1.007 & 0.175 & 1.229 & 0.741 & 0.055 & 0.00 \\
\hline & & $(0.102,0.122)$ & $(1.000,1.066)$ & $(0.162,0.192)$ & $(0.162,0.192)$ & $(0.135,0.99)$ & & \\
\hline \multirow[t]{2}{*}{2} & 33 & 0.124 & 1.007 & 0.194 & 1.256 & 0.870 & 0.032 & 0.00 \\
\hline & & $(0.118,0.131)$ & $(1.000,1.065)$ & $(0.185,0.204)$ & $(1.244,1.269)$ & $(0.527,0.996)$ & & \\
\hline \multirow[t]{2}{*}{3} & 35 & 0.124 & 1.007 & 0.196 & 1.256 & 0.865 & 0.030 & 0.00 \\
\hline & & $(0.116,0.131)$ & $(1.000,1.053)$ & $(0.185,0.207)$ & $(1.241,1.271)$ & $(0.419,0.994)$ & & \\
\hline \multirow[t]{2}{*}{5} & 26 & 0.129 & 1.013 & 0.200 & 1.267 & 0.891 & 0.032 & 0.00 \\
\hline & & $(0.123,0.136)$ & $(1.000,1.109)$ & $(0.190,0.211)$ & $(1.253,1.281)$ & $(0.611,0.993)$ & & \\
\hline \multirow[t]{2}{*}{6} & 34 & 0.161 & 1.007 & 0.249 & 1.331 & 0.858 & 0.047 & 0.00 \\
\hline & & $(0.152,0.168)$ & $(1.000,1.063)$ & $(0.236,0.259)$ & $(1.313,1.345)$ & $(0.544,0.992)$ & & \\
\hline \multirow[t]{2}{*}{7} & 25 & 0.08 & 1.005 & 0.130 & 1.167 & 0.803 & 0.024 & 0.00 \\
\hline & & $(0.072,0.089)$ & $(1.000,1.057)$ & $(0.119,0.142)$ & $(1.151,1.185)$ & $(0.158,0.991)$ & & \\
\hline \multirow[t]{2}{*}{8} & 35 & 0.181 & 1.056 & 0.282 & 1.373 & 0.857 & 0.027 & 0.00 \\
\hline & & $(0.168,0.190)$ & $(1.002,1.206)$ & $(0.264,0.295)$ & $(1.347,1.391)$ & $(0.449,0.992)$ & & \\
\hline \multirow[t]{2}{*}{9} & 29 & 0.087 & 1.005 & 0.136 & 1.180 & 0.912 & 0.017 & 0.00 \\
\hline & & $(0.082,0.092)$ & $(1.000,1.053)$ & $(0.128,0.145)$ & $(1.169,1.192)$ & $(0.655,0.993)$ & & \\
\hline \multirow[t]{2}{*}{10} & 27 & 0.114 & 1.008 & 0.179 & 1.235 & 0.873 & 0.042 & 0.00 \\
\hline & & $(0.107,0.121)$ & $(1.000,1.072)$ & $(0.169,0.190)$ & $(1.222,1.25)$ & $(0.514,0.994)$ & & \\
\hline \multirow[t]{2}{*}{11} & 26 & 0.142 & 1.008 & 0.222 & 1.293 & 0.895 & 0.048 & 0.00 \\
\hline & & $(0.133,0.151)$ & $(1.000,1.074)$ & $(0.209,0.235)$ & $(1.275,1.311)$ & $(0.567,0.996)$ & & \\
\hline \multirow[t]{2}{*}{12} & 27 & 0.153 & 1.290 & 0.238 & 1.315 & 0.816 & 0.034 & 0.00 \\
\hline & & $(0.145,0.161)$ & $(1.063,1.624)$ & $(0.227,0.249)$ & $(1.300,1.331)$ & $(0.469,0.99)$ & & \\
\hline \multirow[t]{2}{*}{13} & 18 & 0.072 & 1.008 & 0.117 & 1.151 & 0.745 & 0.038 & 0.00 \\
\hline & & $(0.064,0.082)$ & $(1.000,1.077)$ & $(0.103,0.133)$ & $(1.134,1.172)$ & $(0.125,0.993)$ & & \\
\hline \multirow[t]{2}{*}{14} & 17 & 0.058 & 1.012 & 0.099 & 1.123 & 0.81 & 0.015 & 0.005 \\
\hline & & $(0.050,0.070)$ & $(1.000,1.115)$ & $(0.085,0.117)$ & $(1.107,1.148)$ & $(0.162,0.993)$ & & \\
\hline
\end{tabular}


TABLE 4. Mean and sample variance of number of unique genotypes identified, by number of markers used.

\begin{tabular}{ccc}
\hline $\begin{array}{c}\text { Number of } \\
\text { markers } \\
\text { used }\end{array}$ & $\begin{array}{c}\text { Mean number of } \\
\text { unique genotypes } \\
\text { identified }\end{array}$ & $\begin{array}{c}\text { Sample variance } \\
\text { of number of } \\
\text { unique } \\
\text { genotypes } \\
\text { identified }\end{array}$ \\
\hline 10 & 110.2 & 7044.3 \\
30 & 303.0 & 2494.1 \\
50 & 345.1 & 225.5 \\
70 & 354.8 & 23.9 \\
90 & 357.2 & 3.4 \\
110 & 357.9 & 0.1 \\
130 & 357.9 & 0.2 \\
\hline 150 & & \\
\hline
\end{tabular}




\section{FIGURES}

A.)
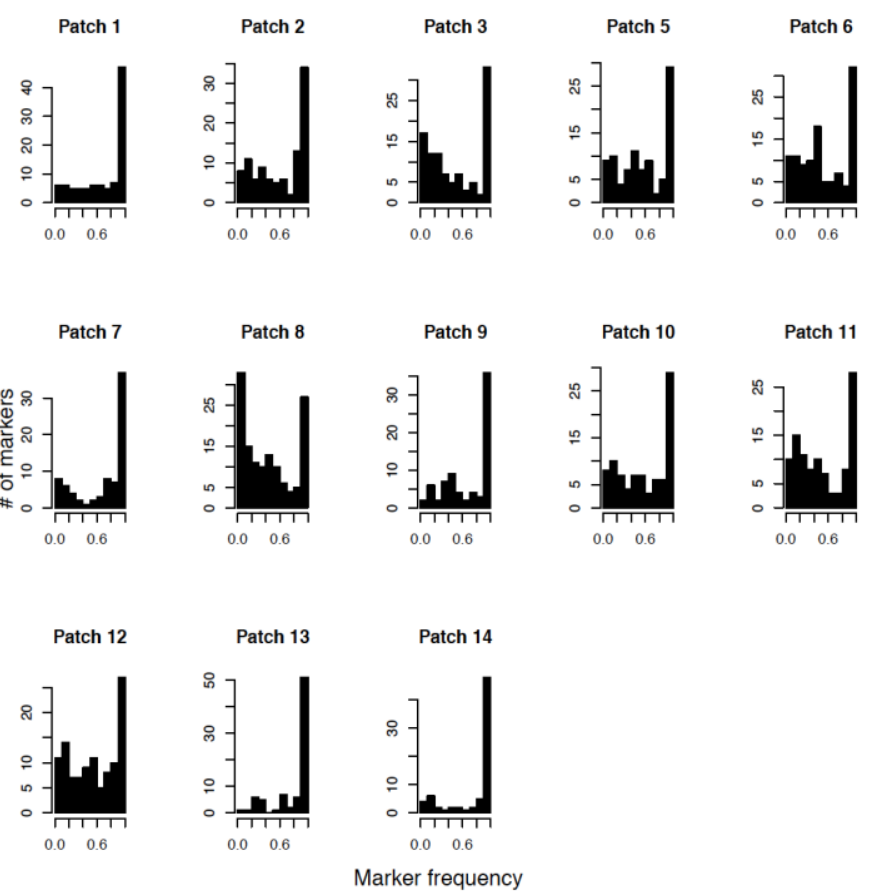

B.)
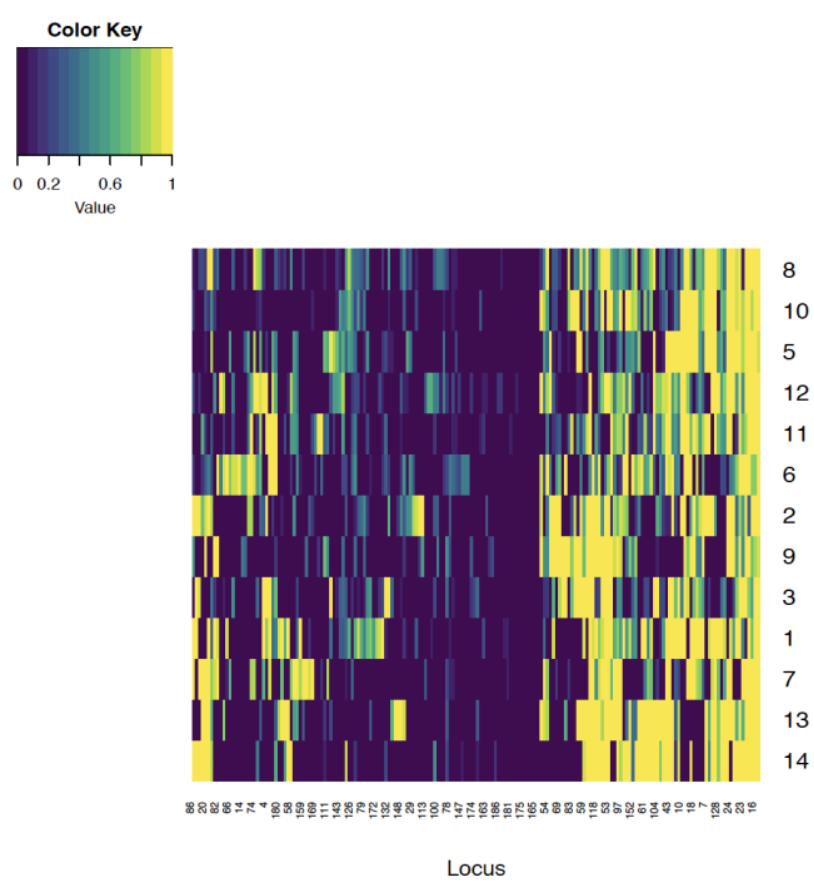

FIGURE 1. A). Total Number ISSR markers that occur in a given frequency, by patch. B.)

Relative frequencies of individuals in each patch carrying at least one copy of each locus. 
A.)

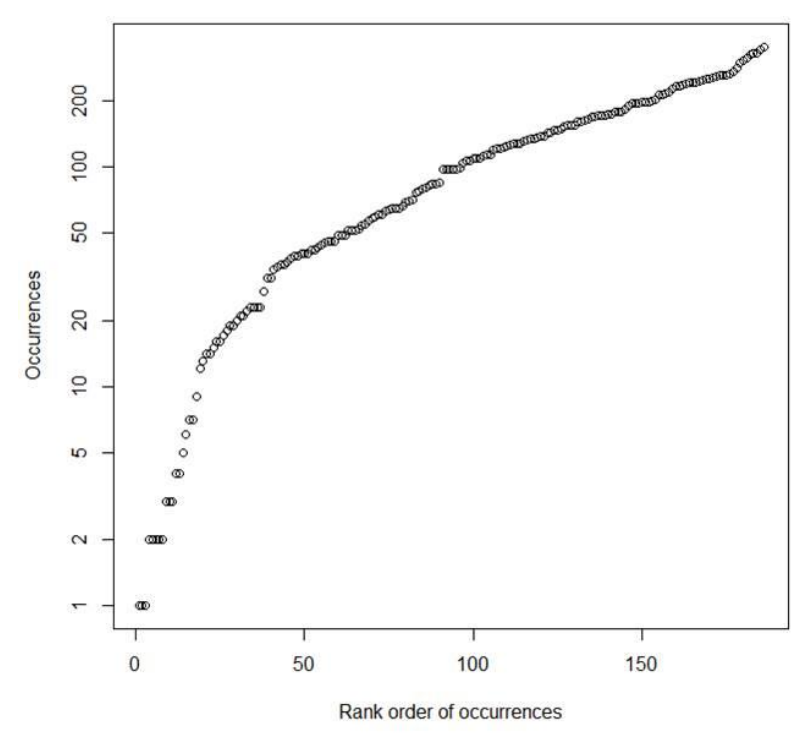

B.)

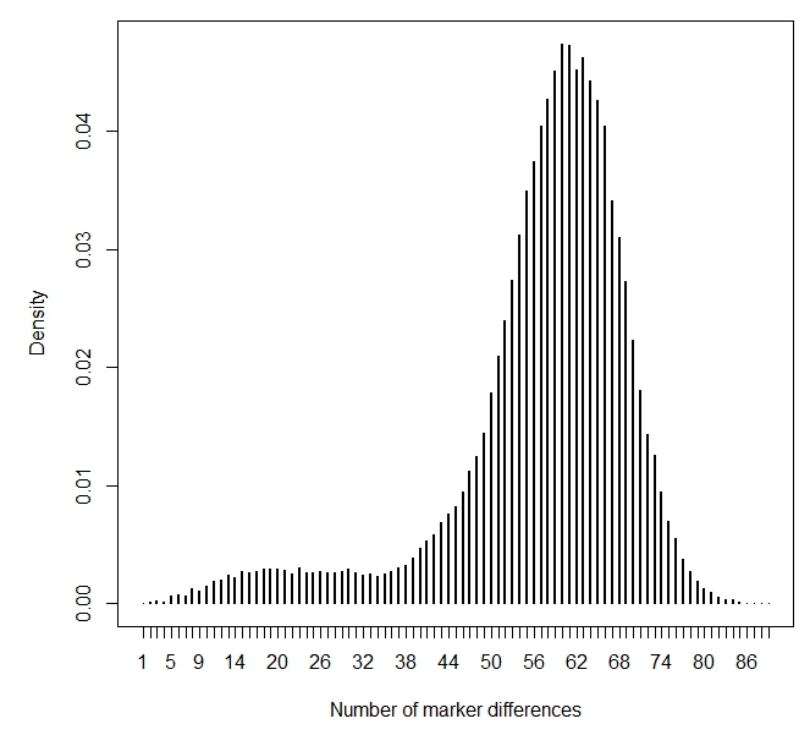

FIGURE 2. Distribution of 186 ISSR markers across the 358 individuals. A.) Number of occurrences of each of the markers (log scale). B.) Comparisons of number of marker differences between pairs of ramets. 


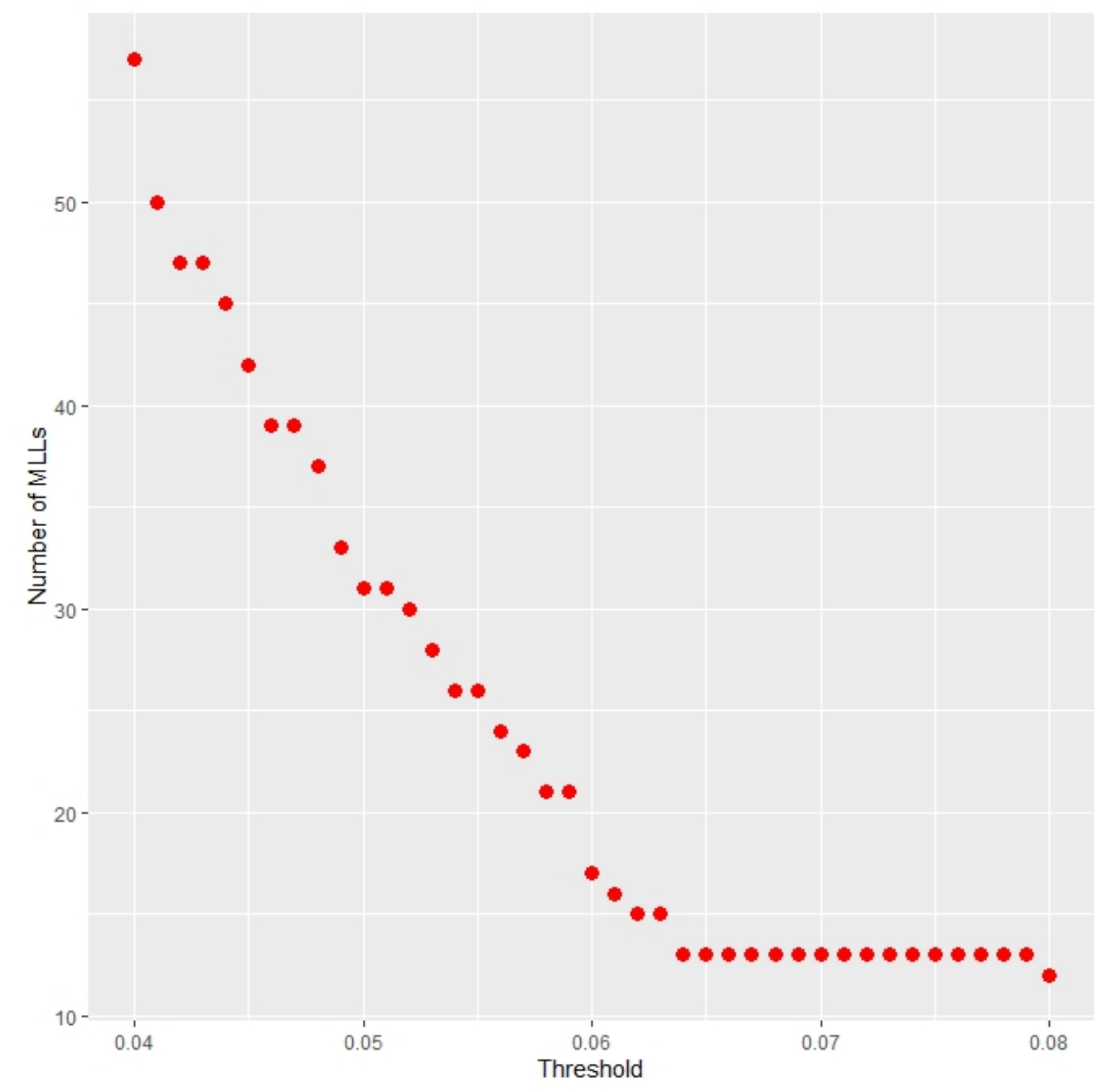

FIGURE 3. Inferred number of multilocus lineages (MLLs) as a function of the threshold

Prevosti distance used to assign ramets to MLLs. For distances less than 0.064 , there is a linear decline in inferred number of multilocus lineages (MLLs) with increasing distance. For thresholds between 0.064 and 0.08 , there is a stable estimate of 13 MLLs, suggesting 0.064 as an appropriate threshold. 

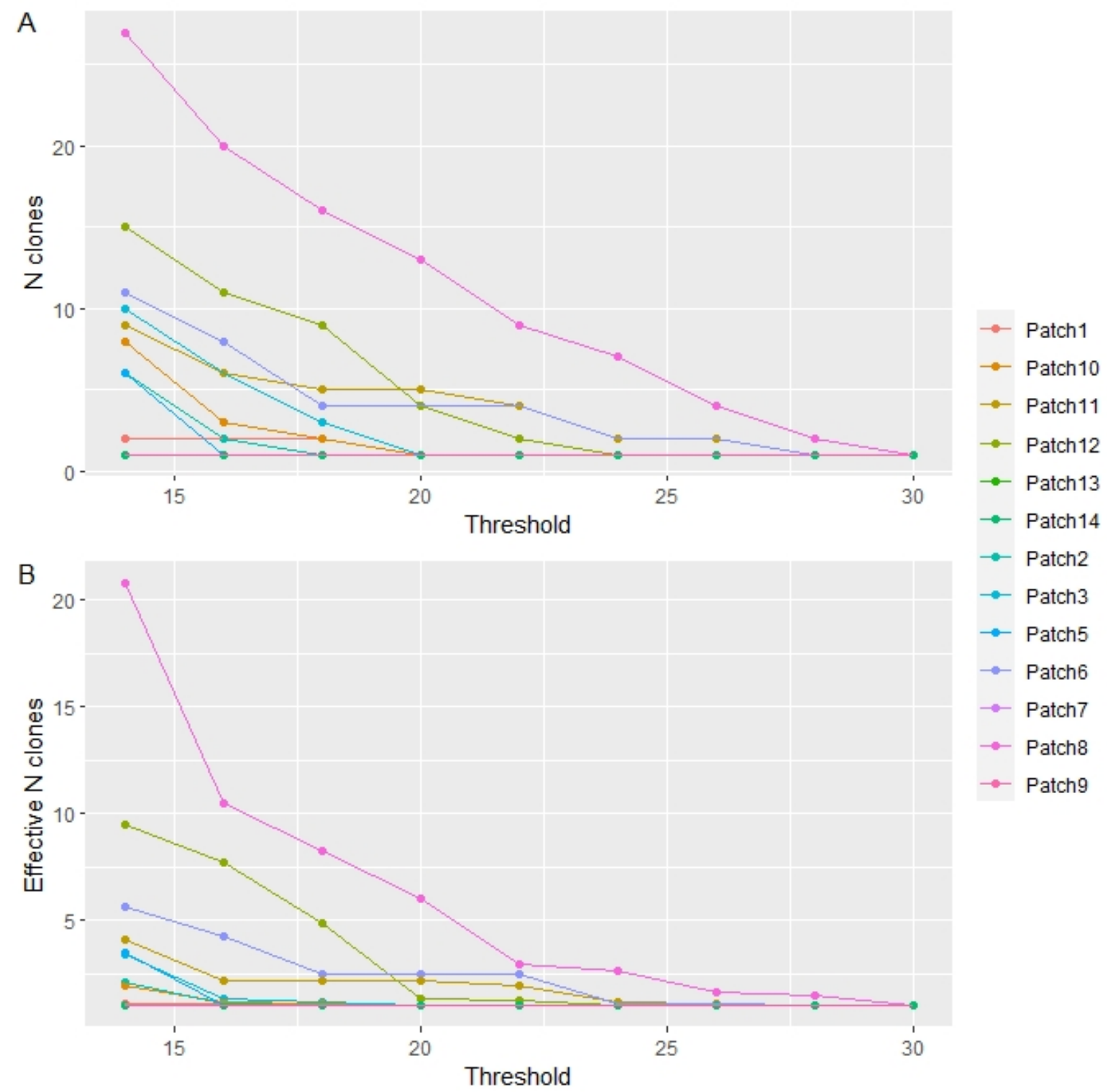

FIGURE 4. Number of MLLs per patch (A) and effective number of MLLs per patch (B), as functions of the threshold number of marker differences used to assign individuals to MLLs. Assignment was made based on the infinite alleles model. 


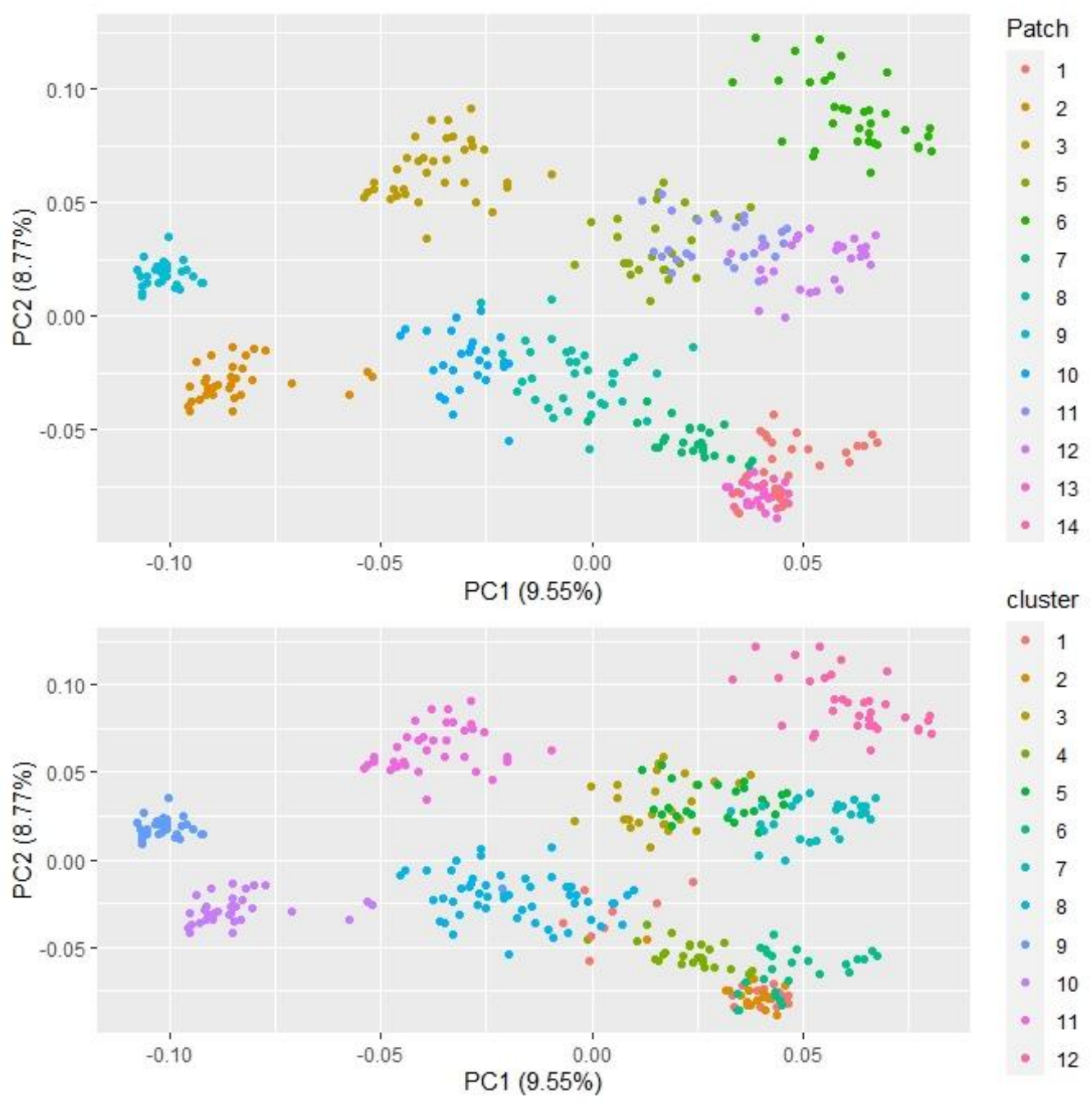

FIGURE 5. Plot of first two principal component scores. Points are labeled with (top) patch number, and (bottom) PCA cluster numbers. 

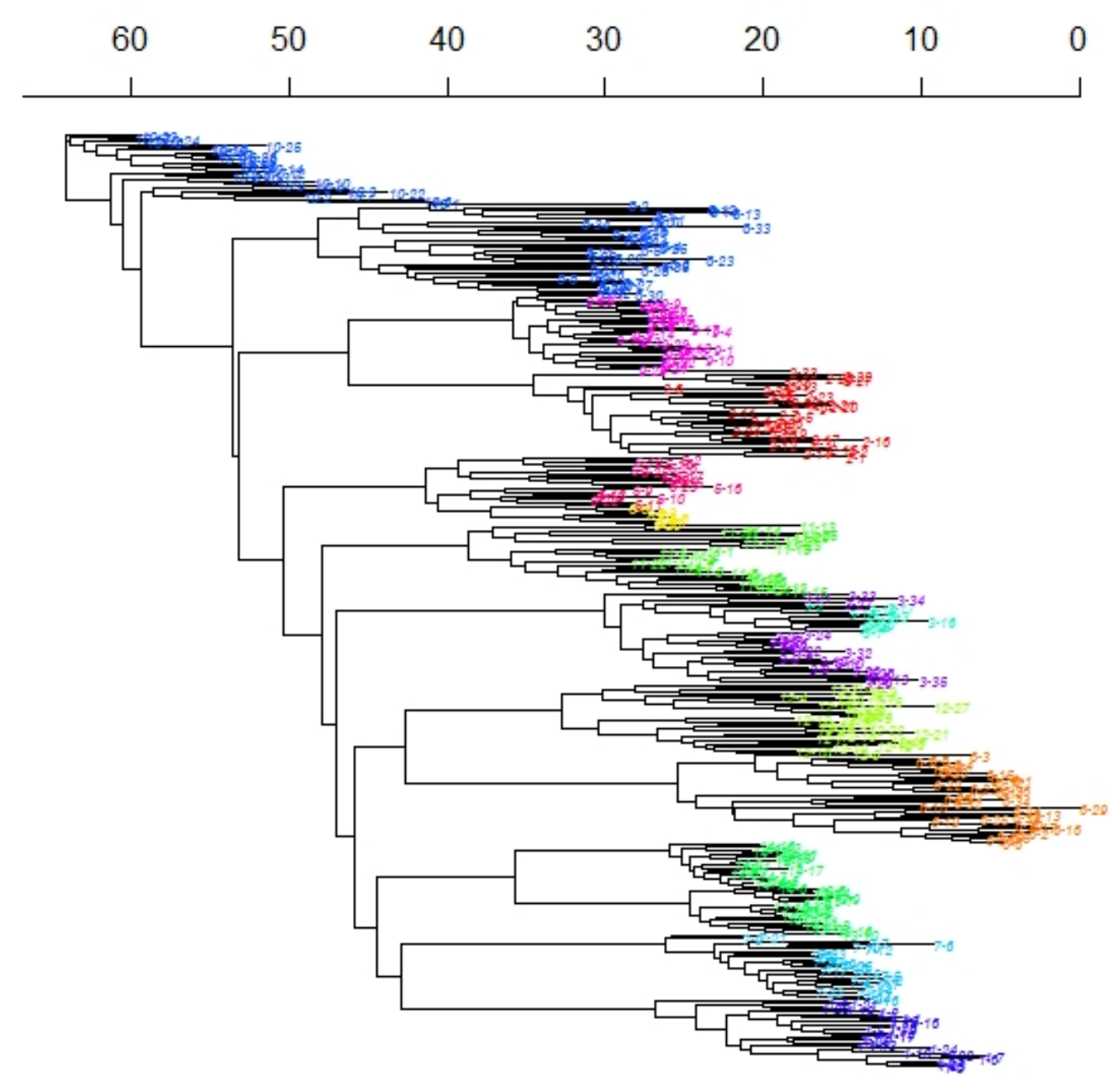

FIGURE 6. Neighbor-joining tree, with branch tips colored by cluster from the cluster analysis. 

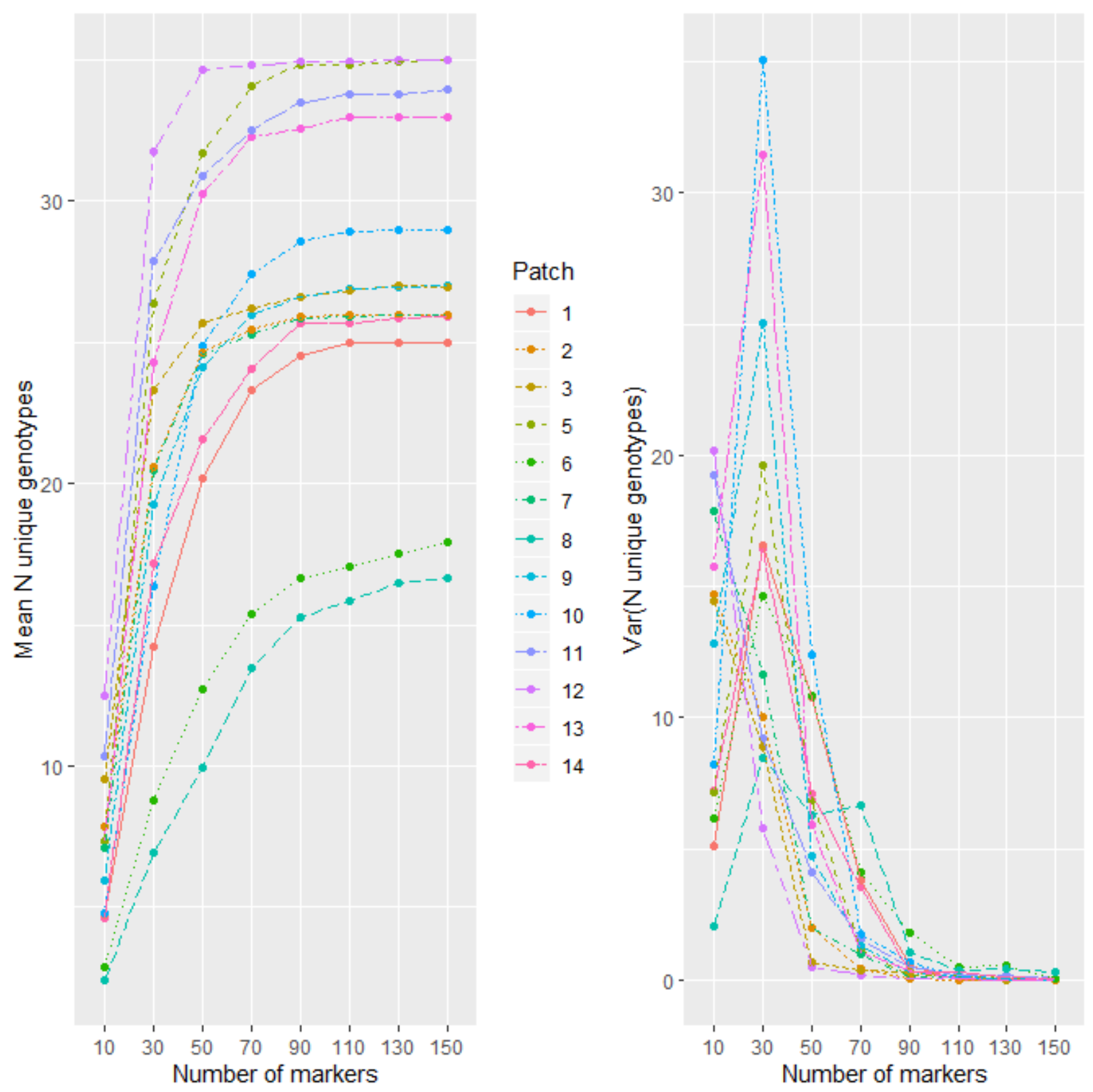

FIGURE 7. The sample means (left) and variances (right) of the number of unique genotypes in each patch, as a function of the numbers of markers used. 